\title{
Detection of ultralow-frequency cavity modes using spacecraft data
}

\author{
C. L. Waters ${ }^{1}$ and K. Takahashi \\ Applied Physics Laboratory, Johns Hopkins University, Laurel, Maryland, USA
}

D.-H. Lee

Department of Astronomy and Space Science, Kyung Hee University, Yongin, Kyunggi, Korea

\section{B. J. Anderson}

Applied Physics Laboratory, Johns Hopkins University, Laurel, Maryland, USA

Received 23 July 2001; revised 3 January 2002; accepted 9 January 2002; published 9 October 2002.

[1] The cold, magnetized plasma in the Earth's magnetosphere supports two ultralowfrequency plasma wave modes. Both these modes may exhibit resonant oscillations in the magnetosphere cavity. Theoretical and numerical studies have predicted the existence of cavity/waveguide resonance modes, yet experimental evidence is sparse. In this paper we detail the expected structure of these modes using both one dimensional (1-D) and three-dimensional (3-D) magnetohydrodynamic (MHD) numerical models. The cavity/ waveguide mode structures are examined in order to develop experimental detection methods suitable for spacecraft electric and magnetic field perturbation data. Cavity mode resonances in the 1-D model suggest a detection method based on wave polarization using the radial $\left(b_{\mathrm{x}}\right)$ and field-aligned $\left(\mathrm{b}_{\mathrm{z}}\right)$ magnetic perturbations. However, when implemented, this method failed to identify cavity/waveguide modes in the magnetic field data recorded by Active Magnetospheric Particle Tracer Explorers/CCE for events that showed pronounced field line resonances in the azimuthal $\left(b_{\mathrm{y}}\right)$ channel. An examination of data from a 3-D MHD numerical simulation showed that the cavity/waveguide resonant signature was identified best in $b_{z}$ component data. Consequently, a wave mode detection method using the $b_{z}$ data from two spatially separated satellites is discussed. Magnetometer data examples from the ISEE 1 and 2 spacecraft show that field line resonances appear in the $b_{\mathrm{y}}$ data even when the coherence length of the $b_{\mathrm{z}}$ data is less than $0.4 R_{E}$. INDEX TERMS: 2149 Interplanetary Physics: MHD waves and turbulence; 2752 Magnetospheric Physics: MHD waves and instabilities; 2772 Magnetospheric Physics: Plasma waves and instabilities; KEYWORDS: ULF waves, cavity modes, magnetosphere

Citation: Waters, C. L., K. Takahashi, D.-H. Lee, and B. J. Anderson, Detection of ultralow-frequency cavity modes using spacecraft data, J. Geophys. Res., 107(A10), 1284, doi:10.1029/2001JA000224, 2002.

\section{Introduction}

[2] The magnetic field of the Earth extends into space to form the magnetosphere within the interplanetary medium. The cold, magnetized plasma in the magnetosphere supports fast (compressional) and the shear Alfven, magnetohydrodynamic (MHD) wave modes [Stix, 1962; Alfvén and Fälthammar, 1963]. These wave modes, in the ultralowfrequency ((ULF) $1-100 \mathrm{mHz}$ ) band, have wavelengths of order the size of the magnetosphere cavity. Their properties are sensitive to the plasma dynamics and geometry of the magnetosphere. The shear Alfven wave energy propagates in the direction of $\pm \mathbf{B}_{0}$, the background magnetic field. For highly reflective conjugate ionospheres, this mode forms

\footnotetext{
${ }^{1}$ Now at School of Mathematical and Physical Sciences and CRC for Satellite Systems, University of Newcastle, Callaghan, New South Wales, Australia.

Copyright 2002 by the American Geophysical Union. 0148-0227/02/2001JA000224\$09.00
}

standing oscillations, known as field line resonances (FLRs) [Obayashi and Jacobs, 1958; Dungey, 1963; Radoski, 1966]. The sensitivity of the shear Alfven mode to plasma mass density allows FLR based methods for remote sensing magnetosphere plasma dynamics [Gul'elmi, 1967; Kitamura and Jacobs, 1968; Waters et al., 1996; Troitskaya, 1997; Waters, 2000]. The fast mode may propagate at arbitrary angles to $\mathbf{B}_{0}$. Resonances of this mode, if detected, could provide information regarding the geometry of the magnetosphere.

[3] Ultralow-frequency, MHD waves are generated around the bow shock region [Tsurutani and Rodriguez, 1981; Hoppe et al., 1981] and magnetopause [Atkinson and Watanabe, 1966; Southwood, 1968] and cross into the magnetosphere [Greenstadt et al., 1983; Engebretson et al., 1987]. In particular, ULF wave activity in the $\sim 15$ to $100 \mathrm{mHz}$ band is known to be associated with small interplanetary magnetic field (IMF) cone angles with frequency proportional to the IMF magnitude [Troitskaya et al., 1971; Arthur and McPherron, 1977; Greenstadt and 
Olson, 1977]. Experimental detection of FLRs is straightforward and FLRs are routinely found from the magnetopause to low latitudes ( $L \sim 1.5$ ), mostly over the dayside magnetosphere [Samson et al., 1971; Orr, 1973; Baransky et al., 1989; Takahashi, 1991; Waters et al., 1991; Anderson and Engebretson, 1995; Menk et al., 2000]. FLR excitation over most of the magnetosphere is generally thought to arise from coupling with fast mode MHD waves that may propagate across the background magnetic field [Chen and Hasegawa, 1974a; Southwood, 1974]. Initially, the favored excitation mechanism for fast mode energy was identified at the magnetopause via the Kelvin-Helmholtz (KH) instability [Tamao, 1966; Chen and Hasegawa, 1974a; Southwood, 1974]. However, the observed correlation of solar wind parameters with ULF wave excitation deep within the magnetosphere [Troitskaya et al., 1971], clear harmonic structure throughout the plasma trough from spacecraft data [Takahashi and McPherron, 1984] and the difficulty in radial propagation for waves generated by the KH mechanism [Southwood, 1968], showed the KH energy source could only be partially responsible.

[4] During the mid 1980s, the role of fast mode waves in the excitation of FLRs was revised. Using the box model of Radoski [1974], Kivelson et al. [1984] modeled resonance of fast mode waves (cavity modes) in the magnetosphere cavity. Subsequent numerical simulations of cavity mode excitation of FLRs showed similar features [Kivelson and Southwood, 1985, 1986; Allan et al., 1986a, 1986b; Inhester, 1987; Zhu and Kivelson, 1988; Krauss-Varban and Patel, 1988; Mond et al., 1990; Lee and Lysak, 1991]. These models are intuitively pleasing, devised only from the geometry of the physical system, simple boundary conditions and a source of ULF wave energy. Consequently, energy transfer from fast mode waves to the excitation of FLRs is well understood, at least in the linear MHD sense and the main features are reproducible in 1-D, 2-D, and 3-D numerical simulations. For $L>6$, the cylindrical model of Allan et al. [1986b] indicate that fast mode resonance frequencies are in the Pc3-4 range $(10-100 \mathrm{mHz})$, higher than the fundamental mode FLR frequencies. The coupling of fast mode resonances to FLRs also provides an explanation for the appearance of FLRs in a magnetized medium that varies continuously with radial distance. Any preferential excitation of an FLR in a thin radial section of the magnetosphere may be attributed to the structure and separation in frequency of cavity resonances.

[5] Observational support for resonance properties of fast mode waves in the magnetosphere is sparse. Some promising evidence comes from ULF waves detected within the plasmasphere. Pi2 pulsations have been interpreted in terms of cavity resonance modes of the plasmasphere [Lin et al., 1991; Sutcliffe and Yumoto, 1991; Itonaga et al., 1992; Allan et al., 1996]. For the more continuous ULF oscillations, the Australian low-latitude magnetometer data have been interpreted in terms of plasmasphere cavity modes in the 20-80 $\mathrm{mHz}$ band [Samson et al., 1995; Waters et al., 2000; Menk et al., 2000]. These appear in the power spectra as 'fine structure' peaks spaced approximately 3 to $4 \mathrm{mHz}$ apart. This frequency separation is consistent with MHD models of plasmasphere cavity mode structures. Further out in the plasma trough, particularly within a few Earth radii of the magnetopause, the experimental evidence is more con- troversial. Sustained oscillations at discrete frequencies of $1.3,1.9,2.3,2.6 \ldots \mathrm{mHz}$ have been associated with fast mode resonances [Mitchell et al., 1990; Ruohoniemi et al., 1991; Samson et al., 1991; Samson and Harrold, 1992; Walker et al., 1992; Fenrich et al., 1995]. These frequencies are so low that the fast mode wave cutoff location appears to prohibit resonance in the magnetosphere, prompting a model that involves the bow shock as an outer boundary [Harrold and Samson, 1992].

[6] The properties of the plasma that supports ULF wave propagation in the magnetosphere are well understood. Therefore, recent MHD wave simulations have focused on devising realistic boundary conditions. Experimental observations of the $1.3,1.9,2.3,2.6 \ldots \mathrm{mHz}$ frequencies motivated altering the nightside magnetosphere boundary to allow waveguide type propagation. In the waveguide model, fast mode energy resonates radially between an outer boundary (bow shock or magnetopause) and an inner turning point while propagating azimuthally [Harrold and Samson, 1992; Wright, 1994]. The most recent boundary condition modifications have returned attention to the role of the $\mathrm{KH}$ instability (magnetosheath flows) and how this may alter ULF wave reflection properties of the dayside boundary [Mann et al., 1999; Mills et al., 1999]. Despite these efforts, it is difficult to reconcile the persistence of FLRs in the plasma trough with an excitation source that depends on a cavity under continual dimensional change.

[7] Spacecraft measurements of ULF wave energy in the plasma trough have failed to identify clear cases of fast mode resonances. This is in stark contrast with the persistence of FLRs regularly identified in the azimuthal magnetic component data [e.g., Takahashi and McPherron, 1984]. After examining data from more than 900 orbits of AMPTE/CCE, Anderson et al. [1989, p. 13,427] state that "compressional and poloidal components exhibit comparatively low-level broadband activity which is typical of toroidal resonance activity observed by AMPTE/CCE ... and that compressional global modes do not appear to play an important role in the majority of locally resonant toroidal pulsations observed." In a detailed study of the intensity of ULF wave power in the outer magnetosphere, Anderson and Engebretson [1995] found that local toroidal FLRs are excited without pronounced compressional wave power and the compressional power does not show prominent peaks at particular frequencies but is more broadband. Furthermore, Anderson and Engebretson [1995] proposed a test for compressional mode driven FLRs that involves examining the radial distance over which the compressional signals are coherent. The results of such a test are reported in the present paper. One possible signature of a fast mode resonance was presented by Kivelson et al. [1997] who observed a constant tone at $24 \mathrm{mHz}$ during a spacecraft radial pass through the plasma trough. This event is discussed later in the present paper.

[8] If FLRs derive their excitation energy from fast mode resonances then these resonances are expected to be easily detected when clear, large amplitude FLRs are observed. Dynamic power spectra of ULF wave data recorded by many spacecraft have shown the ubiquitous nature of FLRs in the plasma trough [e.g., Takahashi et al., 1990; Anderson, 1994]. An example from AMPTE/CCE data, also discussed by Kim et al. [1998], is shown in Figure 1. The three panels 


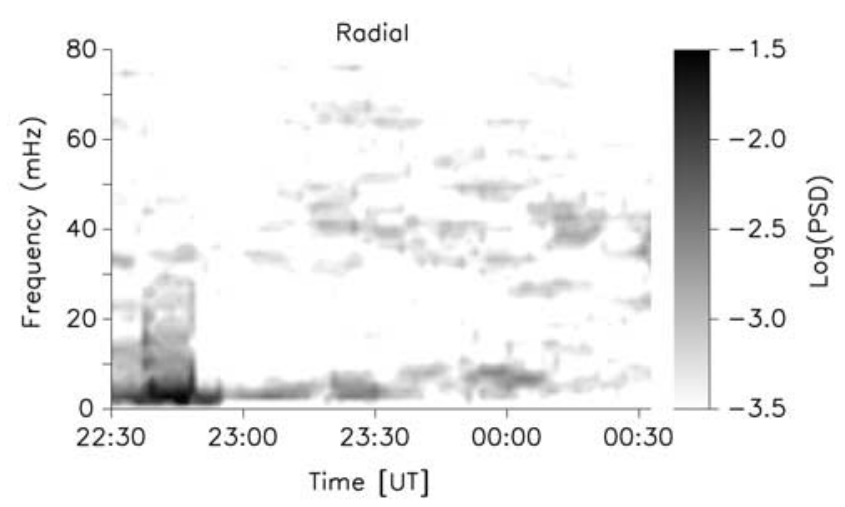

(o)

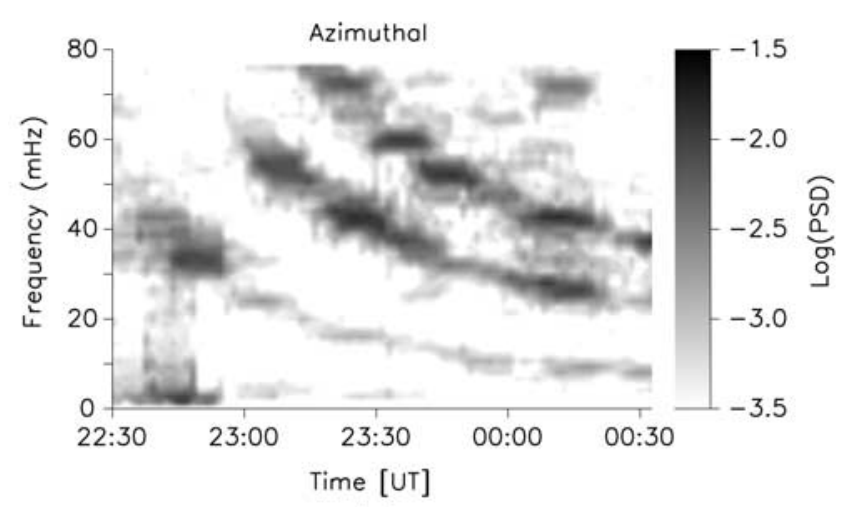

(b)

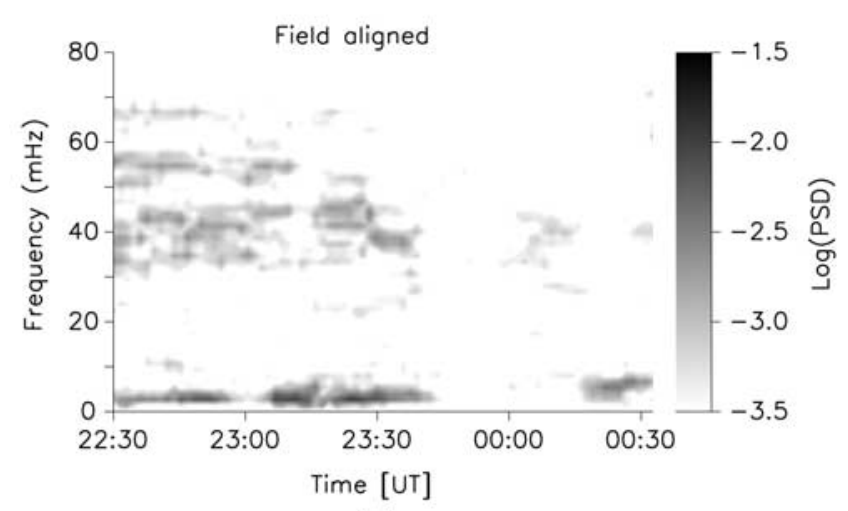

(c)

Figure 1. Power spectra of AMPTE/CCE spacecraft data from an outbound pass (3.3 to $5.9 R_{E}$ ) obtained on 4 November 1984. Note the strong FLR signals in the azimuthal $\left(b_{\mathrm{y}}\right)$ channel. The spacecraft was also moving slightly sunward with MLT varying from 6.6 to 8.2.

show the power spectra of the $b_{x}$ (radial), $b_{y}$ (eastward) and $b_{z}$ (field aligned) magnetic perturbation data. Multiple FLR harmonics are seen in the $b_{y}$ component, $20-80 \mathrm{mHz}$ band with decreasing frequency tones as the spacecraft moves outbound in the plasma trough. In the cavity mode excitation model, enhancements of FLR power represent locations where a cavity resonance and FLR frequencies match. According to computer simulations, cavity resonances would be seen as constant frequency tones with radial distance [e.g., Zhu and Kivelson, 1989; Lee and Lysak,
1999]. Despite the ubiquitous nature of FLRs, routine identification of waveguide or cavity resonances in spacecraft data has not been achieved.

[9] A number of studies have proposed that the FLR energy source may be more impulsive in nature. Allan et al. [1997] suggest that, due to the lack of experimental support, global cavity modes do not appear to be a significant energy source for FLRs in the plasma trough and favor FLR excitation by waveguide modes. Rickard and Wright [1995] simulated DE1 spacecraft trajectories through their model of waveguide generated FLR regions. They modeled time series data that would be detected by a spacecraft sampling a system that was driven by dispersing compressional waves and suggested that the fast mode was best observed near the magnetopause. The essential feature of FLR excitation in these papers involves fast mode waves that travel azimuthally, along the magnetosphere waveguide, close to the magnetopause. A radially traveling spacecraft, away from the magnetopause radial compression source region, would detect pulses of fast mode energy as the wave traveled along the magnetopause. Both these studies employed 2-D models of the magnetosphere. While the waveguide models have some appealing aspects, the lack of experimental support for global cavity modes might be from other factors such as those suggested by Kivelson et al. [1997]. For example, we might have insufficient spacecraft coverage or the data processing methods may need refinement. In the present paper we propose and test alternative data processing methods and analyze data from two radially separated spacecraft, guided by numerical simulations. The simulation data are derived from the 3-D, MHD model described by Lee and Lysak [1999].

[10] There are a number of terms used to describe resonance of the fast mode. Cavity modes occur in MHD models that have highly reflective boundaries while waveguide modes have azimuthal propagation. Waveguide modes with small azimuthal propagation speeds are essentially cavity modes [Lee, 1996]. ULF wave excitation has been studied within the context of quantum mechanics ideas such as tunneling [Zhu and Kivelson, 1989] and the potential barrier [Lee and Kim, 1999]. This latter formulation allowed wave energy leakage through the model boundaries. Resonances in the plasmasphere were denoted virtual resonances and these also appeared in the 3-D treatment by Lee and Lysak [1999]. In the present paper, fast mode ULF waves that exhibit resonant characteristics will be initially denoted as fast mode resonances (FMRs) and details such as reflection properties, tunneling and azimuthal propagation described where appropriate.

[11] In this paper, we present the results of a search for FMRs using spacecraft data. Kivelson et al. [1997] suggested that the identification of FMRs may require "special data processing." In addition to criterion based on ULF wave amplitude, we present the results of data processing based on cross-phase and wave polarization analyses to search for evidence of FMRs in the plasma trough region of the Earth's magnetosphere. These methods also provide a way to estimate the radial distance over which fast mode waves are coherent which should be of the order of a few $R_{E}$ if FMRs are involved [Anderson and Engebretson, 1995]. In order to establish FMR identification criteria, their characteristics are examined using both 1-D and 3-D 


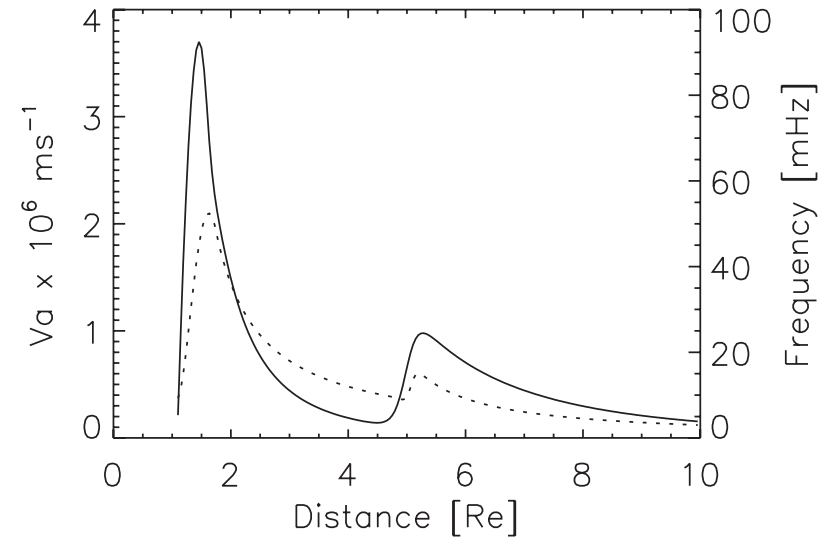

Figure 2. The variation of the Alfven speed (solid) and the frequency of the fundamental FLR (dotted) for the 1-D, numerical model of Waters et al. [2000].

numerical simulations. Since 1-D numerical simulations of the magnetosphere are well within the capabilities of a desktop personal computer, a comparison of 1-D with 3-D simulation results is useful for estimating which features of the 1-D approximations might be retained in the real magnetosphere. The first section of the paper examines the properties of FMRs as revealed by numerical models of ULF wave propagation in the magnetosphere. The second section shows how FMR properties revealed in the simulations may be used to develop suitable data analysis methods. These are then applied to spacecraft measurements of ULF waves to search the plasma trough for evidence of FMRs. In the discussion section, the idea of fast mode excitation of FLRs in the plasma trough is examined in the light of the results from the plasma trough search.

\section{What Should FMRs Look Like?}

\subsection{Cavity Modes in a 1-D, MHD Model}

[12] The simplest numerical simulations of ULF wave activity in the magnetosphere reduce the problem to a single spatial dimension (1-D) approximation consisting of highly reflective inner and outer boundaries, a magnetized cold plasma and energy source. The implementation of 1-D, ideal MHD simulations of the magnetosphere is straightforward and well within present computing capabilities. For a constant, background magnetic field and uniform medium, the polarization properties of both the fast and shear Alfven ULF wave modes are well documented. For ideal, linear MHD, the ULF wave electric field, E, perturbation magnetic field, $\mathbf{b}$, current density, $\mathbf{j}$, and associated perturbation plasma velocity, v, for the fast mode has [e.g., Cross, 1988]

$$
\nabla \cdot \mathbf{E}=0, \quad \nabla \cdot \mathbf{j}=0, \quad \mathbf{E} \cdot \mathbf{b}=0 \quad \nabla \cdot \mathbf{v} \neq 0 .
$$

Most 1-D models of ULF wave propagation and resonance have been formulated assuming a constant background magnetic field. The three-dimensional, linearized MHD equations are simplified by assuming an $\exp \left[\begin{array}{lll}\mathbf{i} & \mathbf{k} & \mathbf{r}\end{array}\right]$ dependence in the field-aligned and azimuthal directions and the system is solved for the radial structure of the perturbation electric and magnetic fields. The model may be time-dependent where the output variables (e.g., perturbation electric and magnetic fields) at each model grid point are given at each time step. Alternatively, the MHD equations can be further simplified by assuming an $\mathrm{e}^{\mathrm{i} \omega \mathrm{t}}$ time dependence. The output parameters are then functions of the frequency, $\omega$ and the simulation describes 'steady state' conditions.

[13] A 1-D model where the background magnetic field and azimuthal and field-aligned wave numbers depend on the radial coordinate was discussed by Waters et al. [2000]. This is a time-independent model where the perturbation quantities assumed an $\mathrm{e}^{\mathrm{i} \omega \mathrm{t}}$ variation. Together with the location of the inner and outer boundaries, the relevant parameters of the modeled magnetosphere are the radial variation of the Alfven speed and the fundamental FLRs (which determines $\mathrm{k}_{\mathrm{z}}$ ). These variables depend on the radial coordinate and are reproduced in Figure 2. For a highly reflective magnetopause, the cavity mode harmonics have a 3 to $4 \mathrm{mHz}$ spacing for frequencies between 20 and 80 $\mathrm{mHz}$. The top three panels of Figure 3 show the radial structure of the $36.8 \mathrm{mHz}$ cavity mode from this model. The fundamental FLR at $36.8 \mathrm{mHz}$ is excited in the plasmasphere at $\sim 2 R_{E}$, as seen in the azimuthal $\left(\mathrm{b}_{\mathrm{y}}\right)$ component. To avoid singularity problems in the vicinity of the FLRs, a small imaginary part was added to the field-aligned wave number, similar to the work of Allan et al. [1986a]. This simulates energy dissipation and may be interpreted as a sink of electromagnetic energy by a resistive ionosphere [Southwood, 1974]. Poynting flux calculations show the expected energy flow from the cavity mode into the FLR. The variation of the radial $\left(b_{x}\right)$ and field-aligned $\left(b_{z}\right)$ components are typical of 1-D, MHD model cavity modes. For this particular model, the turning point associated with $36.8 \mathrm{mHz}$ is located at $2.54 R_{E}$, sunward of the FLR location at $1.98 R_{E}$. In fact, 1-D numerical models show that for a given cavity mode frequency, the location of the FLR at the same frequency is earthward of the cavity mode turning point so that coupling of the fast mode to the FLR occurs where the fast mode is evanescent.

\subsection{Detecting Cavity Modes}

[14] The polarization characteristics of plasma waves are important parameters used in their identification. In wave polarization studies, the combined amplitude and phase information may be represented as a polarization ellipse and described in terms of azimuth and ellipticity. Neglecting the $b_{y}$ component which contains FLR characteristics, the $b_{x}$ and $b_{z}$ data may be treated as a two-component, wave polarization system. The azimuth (measured from the $x$ coordinate) and ellipticity, computed from the $b_{x}$ and $b_{z}$, 1-D model data, for the $36.8 \mathrm{mHz}$ cavity resonance are shown in Figure 3. The maxima in azimuth and ellipticity at the various radial locations suggests a possible cavity mode detection procedure based on wave polarization properties.

[15] For multichannel data that contains a signal exhibiting a particular azimuth and ellipticity (i.e., polarization state, $u$ ) a normalized detector for a particular polarization state may be defined as [Olson and Samson, 1979; Samson and Olson, 1981]

$$
\mathrm{D}=\frac{\mathrm{u}^{+} \mathrm{Su}}{\operatorname{Tr}[\mathrm{S}]},
$$

where $\mathrm{S}$ is the spectral matrix. The polarization state, $u$, is expressed as a Jones' vector (complex), a formulation 
$\left|b_{x}\right|$, real $\left(b_{x}\right)$, phase $\left(b_{x}\right)$

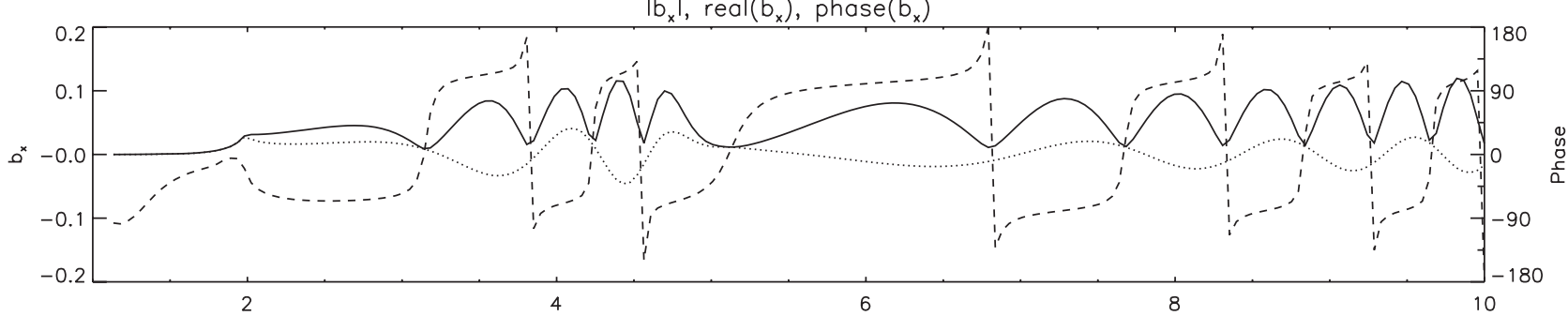

$\left|b_{y}\right|$, real $\left(b_{y}\right)$, phase $\left(b_{y}\right)$

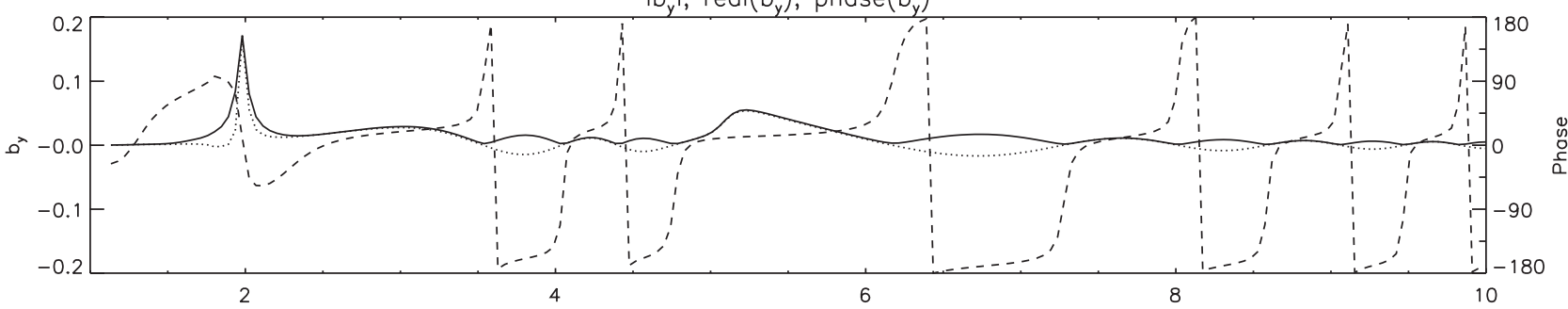

$\left|b_{z}\right|$, real $\left(b_{z}\right)$, phase $\left(b_{z}\right)$
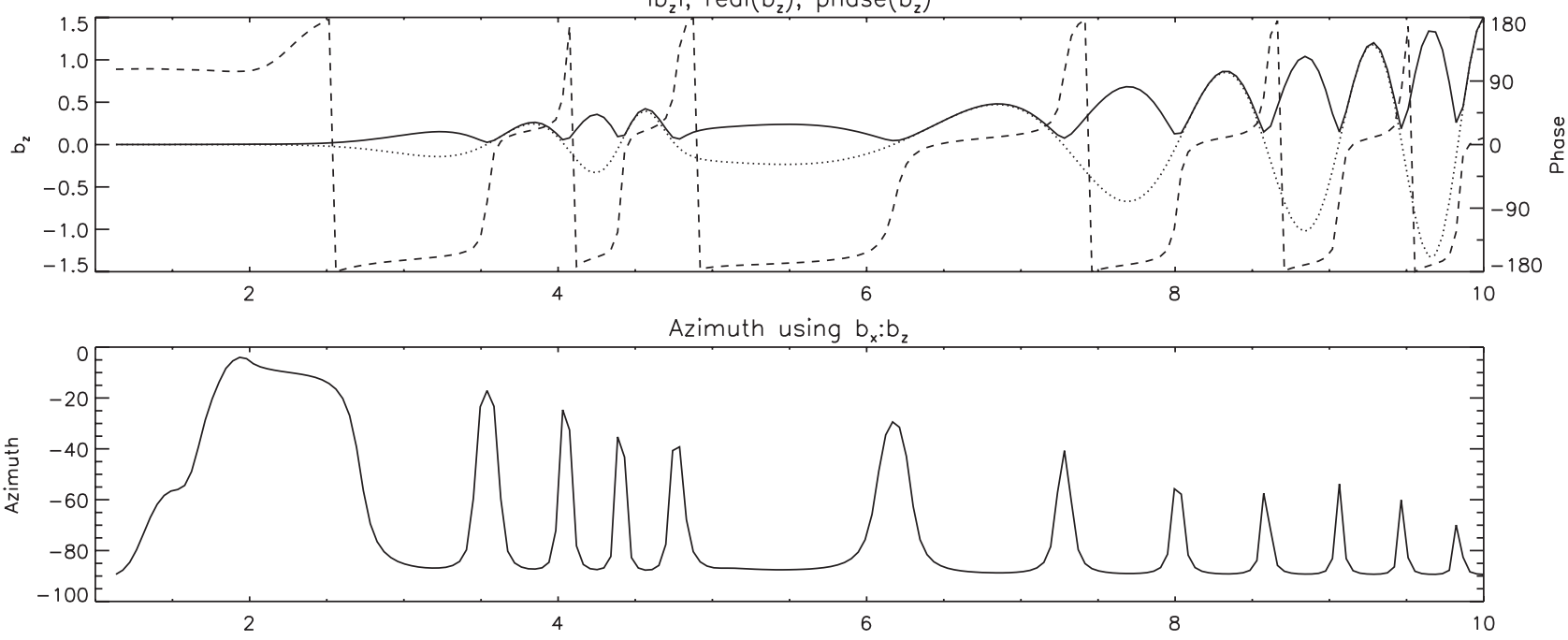

Ellipticity using $b_{x}: b_{z}$

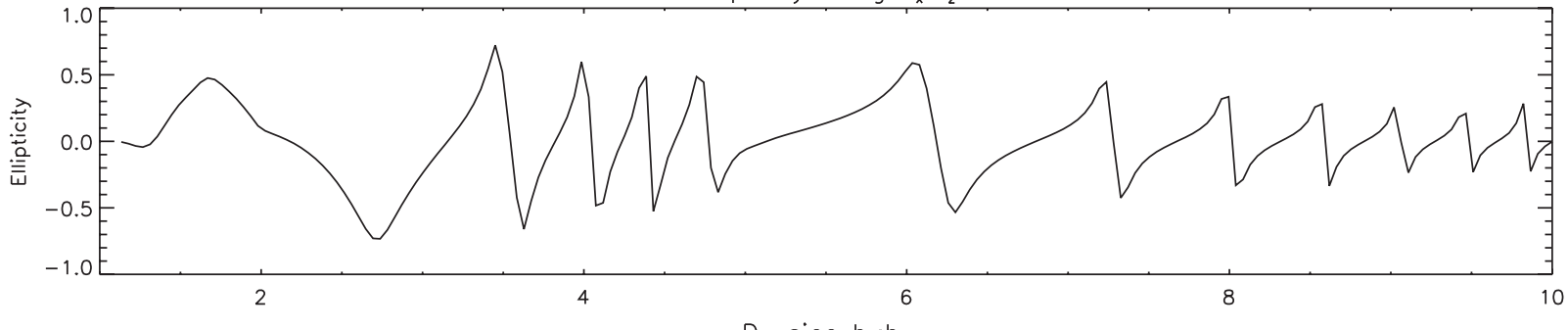

D using $b_{x}: b_{z}$

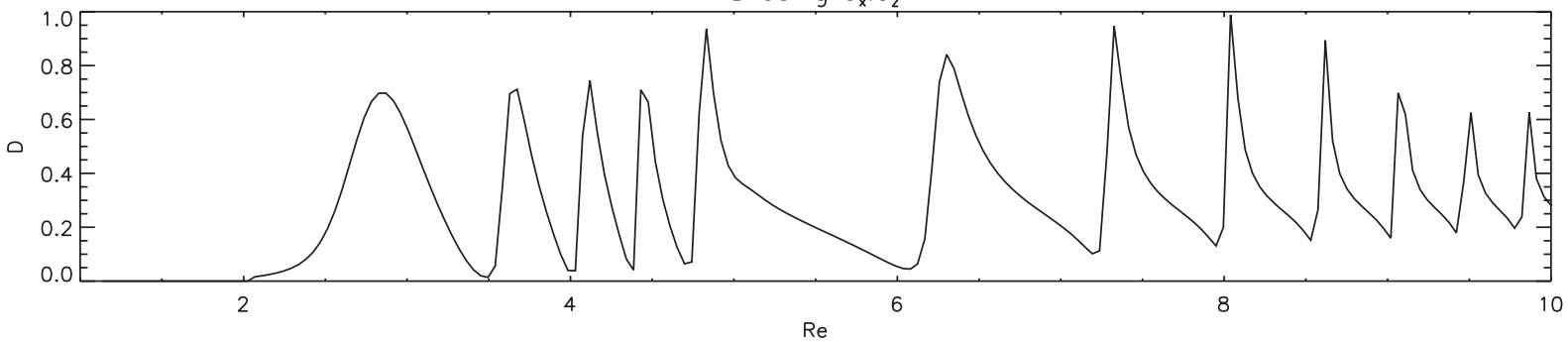

Figure 3. The radial variation of the $36.8 \mathrm{mHz}$ cavity resonance from the 1-D model. The absolute value (solid), real part (dotted) and phase (dashed) of the magnetic field are shown in the top three panels. Note the FLR in $\mathrm{b}_{\mathrm{y}}$ at $\sim 2 R_{E}$. The azimuth, ellipticity, and D (equation (2)) were calculated from the $\mathrm{b}_{\mathrm{x}}$ and $b_{\mathrm{z}}$ data. 
common in optics [e.g. Collet, 1993]. For example, Olson and Samson [1979] discuss a circularly polarized signal at frequency, $\omega_{0}$, in a right-hand sense about the $Z$ axis and confined to the $X Y$ plane. This signal is represented by the vector

$$
\frac{1}{\sqrt{2}}\left[e^{i \omega_{0} t}, e^{i\left(\omega_{0} t+\pi / 2\right)}, 0\right]^{T}
$$

The Fourier transform of equation (3) at frequency $\omega_{0}$ is

$$
\frac{1}{\sqrt{2}}[1, i, 0]^{T}
$$

the Jones' vector representation of the polarization state. For experimental input data, the spectral matrix, $\mathrm{S}$ is Hermitian so $\operatorname{Tr}[\mathrm{S}]$ represents the total signal power (at some frequency) and therefore $0 \leq \mathrm{D} \leq 1$. In practice, the parameter $\mathrm{D}$ is raised to some power (usually 4), analogous to the "order" of a filter. The value of D in (2) is the normalized "expectation value of the power level contained by the data represented by $\mathrm{S}$ in the state $u$ " [Olson and Samson, 1979]. It has a maximum value of unity when the spectral matrix $\mathrm{S}$ is formed from noise-free data in the polarization state, $u$ [see Olson and Samson, 1979, Figure 1].

[16] To develop polarization detection parameters for FMRs, the polarization properties of the $b_{x}$ and $b_{z}$ data from the 1-D model were examined. A similar process for ULF waves in the inner magnetosphere is illustrated by Kim and Takahashi [1999]. Figure 3 shows that zeros in the magnitude of $b_{z}$ correspond with maxima in azimuth. The $b_{x}, b_{z}$ derived polarization state, varies slightly at each azimuth maximum. A typical value (taken at $8 R_{E}$ ) is the complex vector, $u=[(-0.4,0.3 i),(0.8,0.0 i)]$ and the values for $\mathrm{D}$ in equation (2) using the 1-D model, $b_{\mathrm{x}}$ and $b_{z}$ data are shown in the bottom panel of Figure 3. Note that $\mathrm{D} \sim 1$ for $8 R_{E}$. Therefore, on the basis of the 1-D model, around $40 \mathrm{mHz}$, we would expect to see this unique, polarization at about seven radial locations in the plasma trough.

[17] The FMR polarization detection process described above was applied to the radial and compressional components of the AMPTE/CCE magnetometer data shown in Figure 1. This analysis failed to show any evidence of FMRs in the data. The data from the 1-D model and therefore the chosen polarization reflect the large $b_{z}$ amplitude compared with $b_{x}$. However, Figure 1 is typical of AMPTE/CCE data in that the larger amplitude is generally found in the $\mathrm{b}_{\mathrm{y}}$ component [Anderson and Engebretson, 1995]. Furthermore, there is a distinct lack of $b_{z}$ power after 2330 UT. This does not necessarily imply that FMRs do not exist in the data. The relative positions of FMR turning points and FLR locations means that evanescent fast modes couple to the FLRs. Perhaps the $\sim 3 R_{E}$ extent of the AMPTE/CCE data is insufficient to capture the non evanescent part of FMRs. The failure of the polarization detection method might also be due to the representation of the actual magnetosphere as a perfectly reflective, idealized 1-D MHD system. Therefore, FMRs in a 3-D numerical model of the magnetosphere were exam-

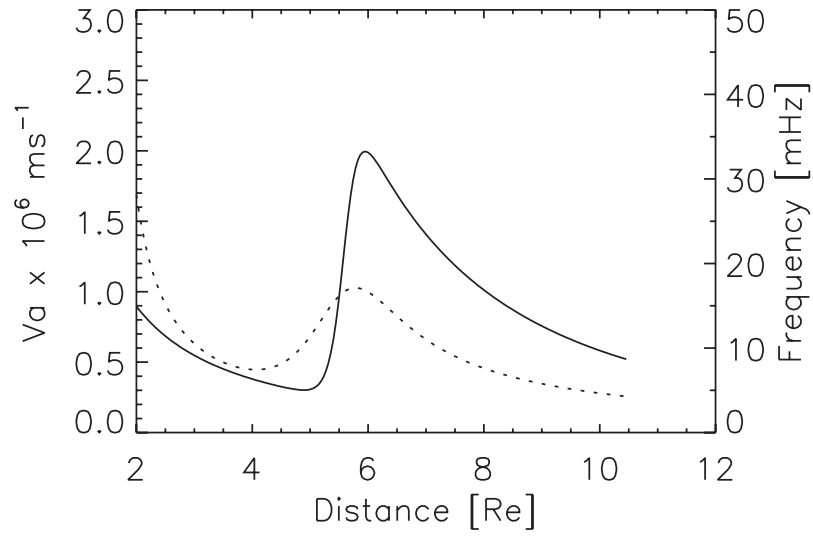

Figure 4. The variation of the Alfven speed (solid) and the frequency of the fundamental FLR (dotted) for the 3-D, numerical model of Lee and Lysak [1999].

ined. Spacecraft data obtained over larger radial distances are examined later.

\subsection{FMRs in a 3-D MHD Model}

[18] The perfectly reflecting outer boundaries have been removed in the 3-D, MHD model of Lee and Lysak [1999]. The linearized, ideal MHD wave equations are solved in a dipole geometry with the inner and outer dayside boundaries at 2.0 and $10.5 R_{E}$, respectively. The variation of the Alfven speed with distance in the equatorial plane is reproduced here as Figure 4 and includes a plasmapause around $5.5 R_{E}$. The magnetopause boundary conditions describe partial wave reflection on the dayside diminishing to zero wave reflection in the nightside, allowing wave energy to propagate out of the simulation. Perfectly reflecting boundaries were assumed at the ionosphere (at $0.5 R_{E}$ ). The model was initialized by a radially directed pulse in the magnetotail, symmetric with respect to the equator as illustrated in Figure 2 of Lee and Lysak [1999]. The symmetric nature of the pulse means that the fundamental and odd FLR harmonics are excited.

[19] Output from the 3-D simulation is in the form of time series, with a resolution of $4 \mathrm{~s}$, of the electric and magnetic field perturbations at all grid locations in the model. Our $\left(b_{x}, b_{y}, b_{z}\right)$ coordinate system corresponds with the $\left(\mathrm{b}_{\nu}, \mathrm{b}_{\phi}, \mathrm{b}_{\mu}\right)$ dipole system of Lee and Lysak [1999]. The excitation pulse began at $t_{0}$ and lasted $60 \mathrm{~s}$. The time series analyzed here were from $t_{0}+400$ to $t_{0}+2448 \mathrm{~s}$. Since AMPTE/CCE trajectories are within $\sim 10^{\circ}$ of the magnetic equator, the data from the equatorial grid points along the 1100 MLT meridian of the 3-D model were initially used. Figure 3 of Lee and Lysak [1999] shows FMR structure in the field-aligned component. Due to the non-perfect reflection at the outer boundary, these modes were called "virtual resonances."

[20] The first step in identifying FMRs in the 3-D simulation was to transfer the time series, from selected grid locations, into the frequency domain using a 512 point FFT. This essentially shifts the interpretation of the data to the time-independent view where the spectral components are assumed constant with time. The amplitude spectra from each grid point along a line slightly off the equatorial plane at MLT $=1100$ were averaged with 

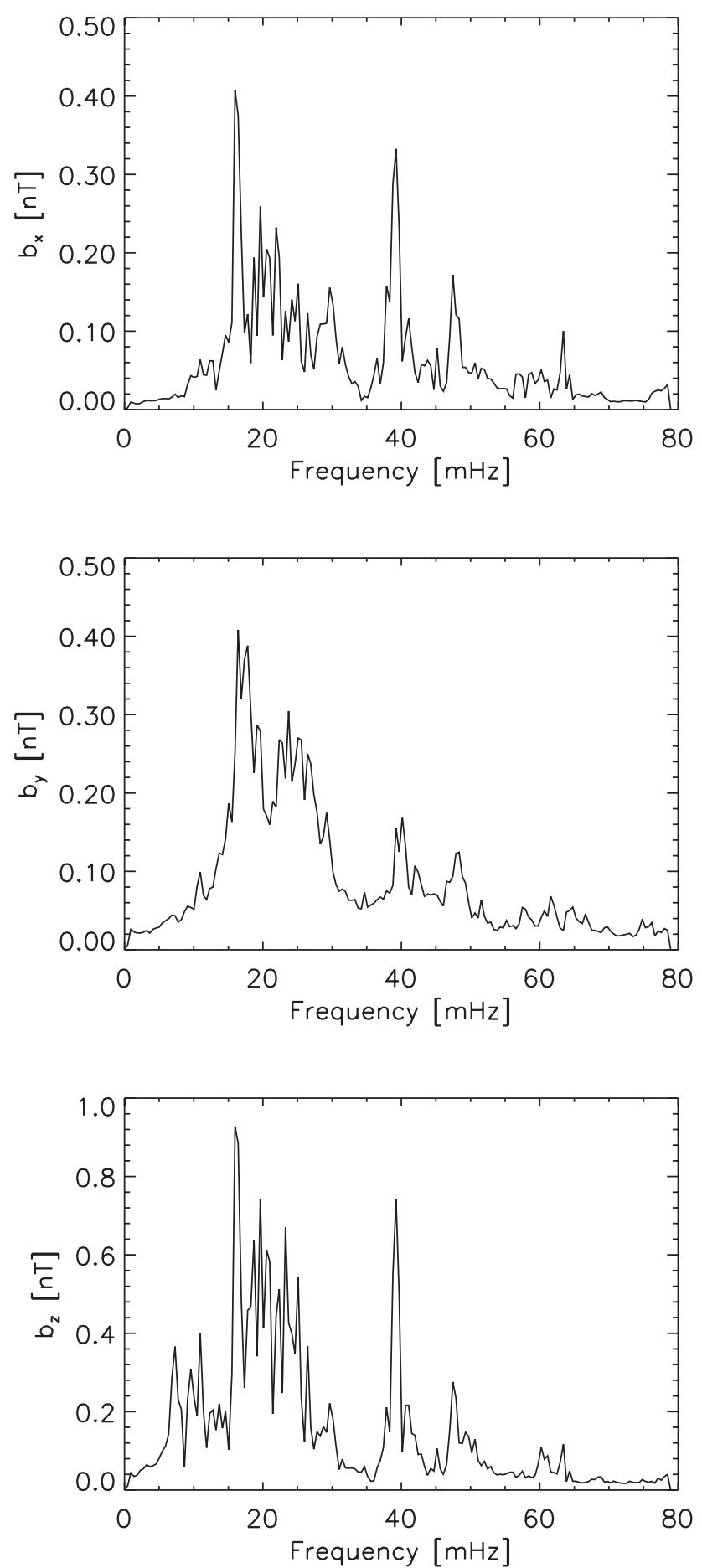

Figure 5. The average of the Fourier transform, amplitude spectra over 6-10.5 $R_{E}$ of the 3-D numerical model data. The data were taken from grid locations close to the equatorial plane along a line at 1100 MLT. The peak in $b_{z}$ at $39 \mathrm{mHz}$ is an FMR.

the result shown in Figure 5. The average $b_{x}$ and $b_{z}$ spectra are quite similar. However, the peaks in the $b_{z}$ component were used to identify the FMR frequencies for reasons that will become apparent below. Each frequency was examined in terms of the radial variation of both the amplitude and phase of $b_{z}$.
[21] The spatial structure of the $39 \mathrm{mHz}$ FMR in the 3-D MHD simulation is shown in Figure 6. The nodes in $b_{z}$ are a feature common with the 1-D results. However, a number of differences with the 1-D magnetic data in Figure 3 are immediately obvious. For example, the amplitude ratio of the compressional to radial perturbations is $\sim 10$ for the cavity resonances in the 1-D model while in the 3-D model, the ratio is much smaller at $\sim 1.5$. Direct amplitude comparisons between the models are not possible as (1) the excitation methods are different, (2) the 3-D model is a time-dependent model and (3) absolute amplitudes in the 3-D model cannot be interpreted quantitatively as discussed by Stellmacher et al. [1997]. The resonance behavior is quite clear in $b_{z}$ with 2.5 wavelengths in the plasma trough for the 39 $\mathrm{mHz}$ resonance (Figure 6). In the 1-D model, there are many more (7.5) wavelengths at the similar frequency of $36.8 \mathrm{mHz}$. Since 1-D simulations are much more accessible in terms of computing resources, it is worth exploring any major differences between 1-D and 3-D simulations.
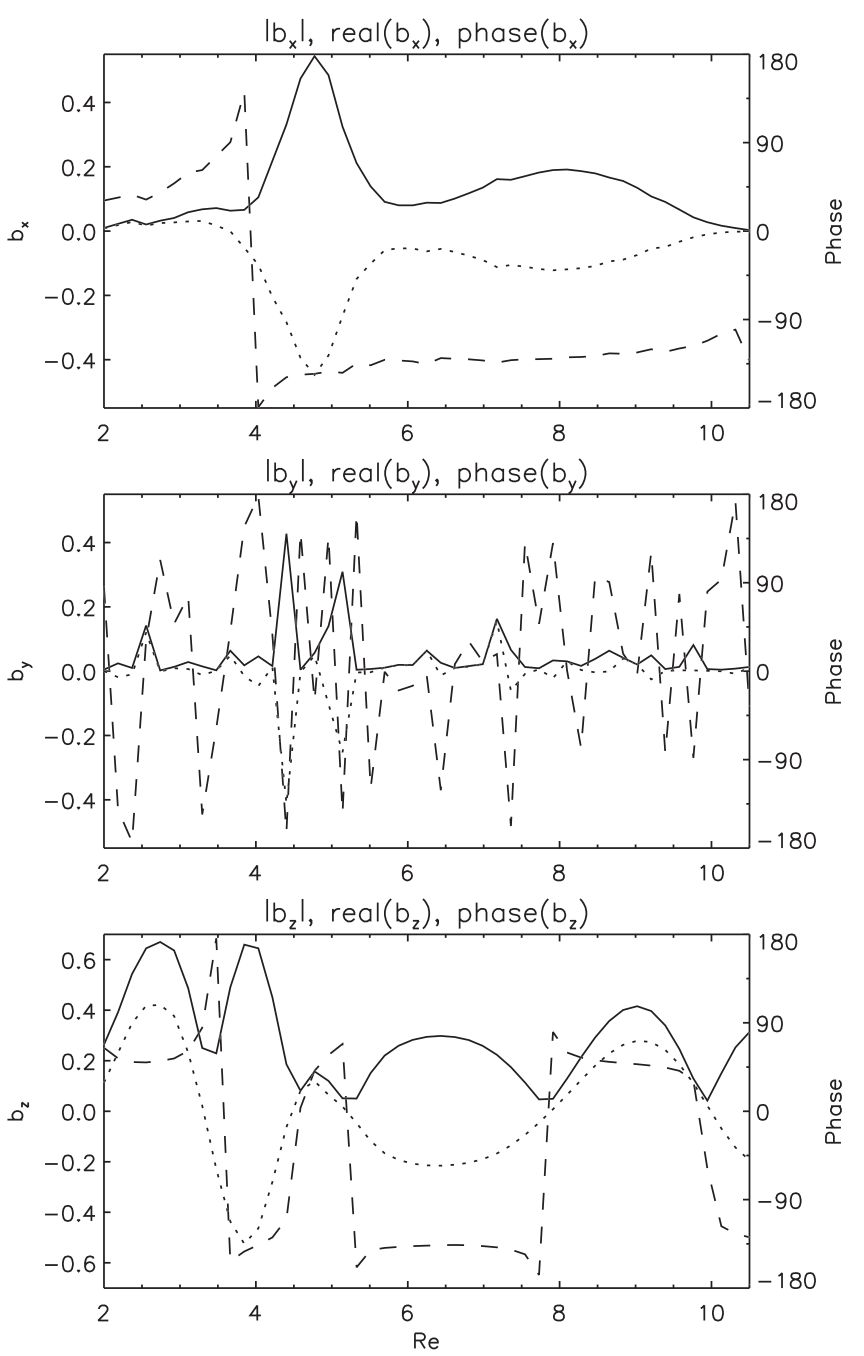

Figure 6. The radial variation of the $39 \mathrm{mHz}$ FMR from the 3-D numerical model. The absolute value (solid), real part (dotted) and phase (dashed) of the magnetic field data were taken from grid locations close to the equatorial plane along a line at 1100 MLT. The $b_{z}$ data show the FMR is well formed in both the plasma trough and the plasmasphere. 

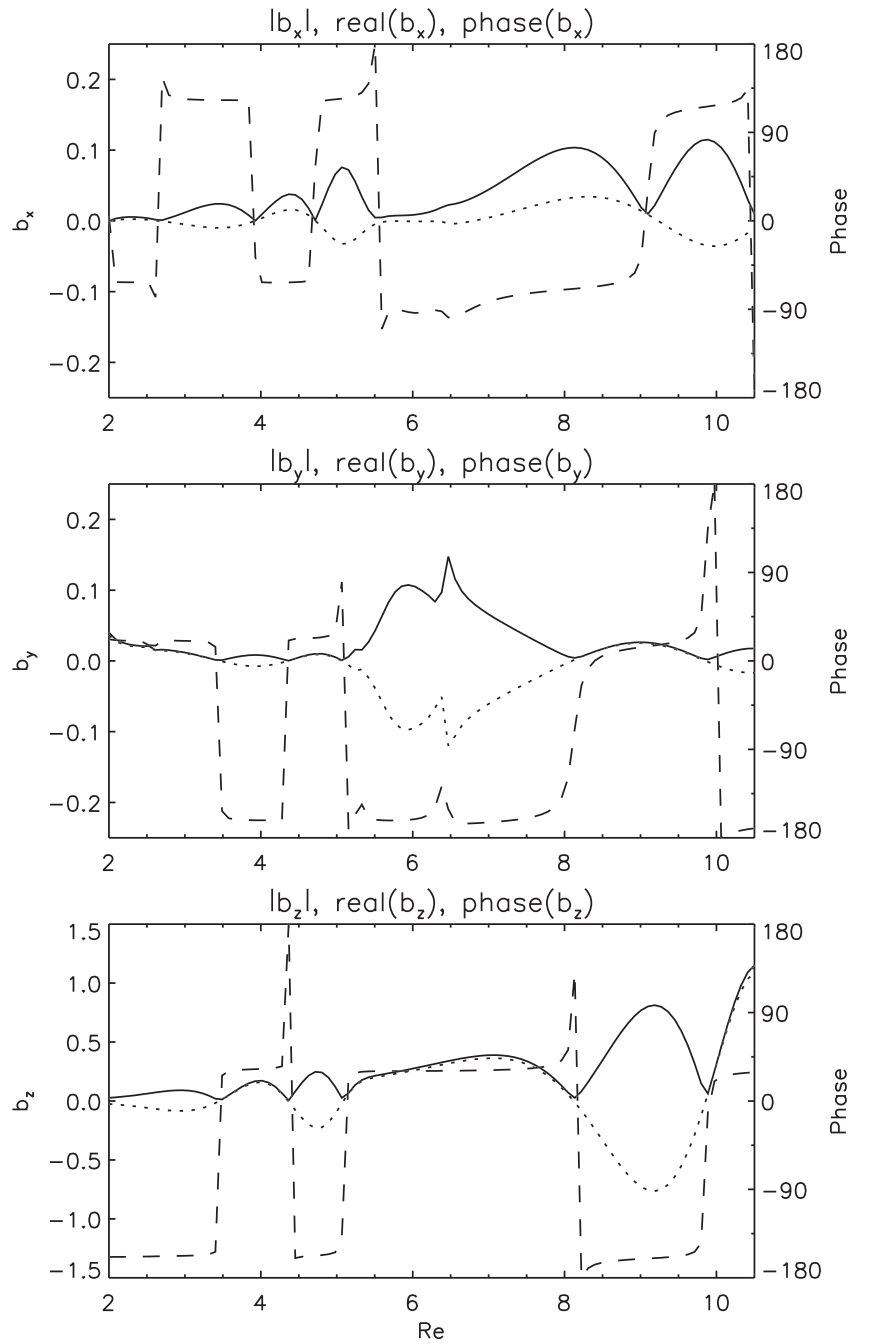

Figure 7. The radial variation of the $35.8 \mathrm{mHz}$ cavity resonance from the 1-D model that used the Alfven speed and FLR variation of the 3-D model (Figure 4). The absolute value (solid), real part (dotted) and phase (dashed) of the magnetic field show resonance characteristics in $b_{x}$ and $b_{z}$. The peak in $\mathrm{b}_{\mathrm{y}}$ near $6.5 R_{E}$ is the third harmonic FLR.

[22] The 1-D MHD model was modified to duplicate parameters used in the 3-D simulation. The inner and outer boundaries in the 1-D model were altered to 2.0 and $10.5 R_{E}$ respectively. The variation of the Alfven speed in the equatorial plane used in the 3-D model (Figure 4) replaced the original 1-D model Alfven speed profile. Finally, the fast mode wave turning points in the 1-D model depend on the FLRs through the field-aligned wave number, $\mathrm{k}_{\mathrm{z}}$. The radial variation of the fundamental FLRs was determined from the 3-D model, $b_{y}$ data. This was used to calculate $\mathrm{k}_{\mathrm{z}}$ for the 1-D model using $k_{\mathrm{z}}=\left[\omega_{\mathrm{FLR}}\right] / \mathrm{v}_{\mathrm{A}}$, where $\omega_{\mathrm{FLR}}$ is the fundamental FLR. Higher-order odd harmonics were generated using odd integer multiples of $\mathrm{k}_{\mathrm{z}}$.

[23] The 3-D model showed an FMR at $39 \mathrm{mHz}$ (Figures 5 and 6) for data taken from the radial line of grid points slightly off the equatorial plane at 1100 MLT. The closest cavity mode in the modified 1-D model was $35.8 \mathrm{mHz}$, and its radial structure is shown in Figure 7.
The number of nodes of $b_{z}$ has decreased to about the same as the 3-D model, $39 \mathrm{mHz}$ FMR. The turning point for the 1-D model, $35.8 \mathrm{mHz}$ FMR is located at $6.0 R_{E}$ for $\mathrm{k}_{\mathrm{z}}=$ $\left[\omega_{\mathrm{FLR}}\right] / \mathrm{v}_{\mathrm{A}}$ and at $6.5 R_{E}$ for $\mathrm{k}_{\mathrm{z}}=3\left[\omega_{\mathrm{FLR}}\right] / \mathrm{v}_{\mathrm{A}}$. The $35.8 \mathrm{mHz}$ fundamental FLR location is very close to the inner boundary $\left(2 R_{E}\right)$ while the $35.8 \mathrm{mHz}$, third harmonic FLR location is $6.4 R_{E}$. The model run for $\mathrm{k}_{\mathrm{z}}=3\left[\omega_{\mathrm{FLR}}\right] /$ $\mathrm{v}_{\mathrm{A}}$ showed a typical FLR signature in $\mathrm{b}_{\mathrm{y}}$ around $6.4 R_{E}$, similar to the amplitude and phase variation seen in Figure 3 around $\sim 2 R_{E}$. While the 1-D model, $b_{z}$ data showed similar properties compared with the 3-D simulation, there are a number of differences. For example, the 3-D simulation results (Figure 6) show increased $b_{z}$ amplitude in the plasmasphere compared with the plasma trough, while the 1-D model data show a reduction in the amplitude of $b_{z}$ in the plasmasphere. The differences in $b_{y}$ stem from the number of FLRs included in the respective models. Some differences are expected from field line curvature effects in the 3-D model, particularly for off equatorial cuts and the differences in azimuthal variation $\left(\mathrm{k}_{\mathrm{y}}\right.$ in the 1-D model). However, the variation of $b_{z}$ near the equatorial plane along the 1100 MLT line in the 3-D model is similar to the 1-D model results for $b_{z}$.

[24] In the search for cavity resonances using wave polarization properties, the most important difference between the 1-D and 3-D model results is the lack of resonance properties in the 3-D model, $b_{x}$ data. For a 1-D cavity model using a "box" magnetosphere with zero fast mode coupling to the shear Alfven mode $\left(\mathrm{k}_{\mathrm{y}}=0\right)$, both $\mathrm{b}_{\mathrm{x}}$ and $\mathrm{b}_{\mathrm{z}}$ should show standing wave behavior [Kim and Takahashi, 1999]. For all FMR frequencies identified from Figure 5 , the $b_{z}$ perturbations showed typical node/antinode structure with phase shifts across the nodes, similar to Figure 6. No such pattern was evident in the $b_{x}$ (radial) data. This result was actually found by a failure to identify any unique $b_{x}: b_{z}$ wave polarization at the FMR frequencies identified from Figure 5. If we trace along a field line, then $b_{x}$ shows an antinode where $b_{z}$ has a node. However, the radial dependence of the relationship between $b_{x}$ and $b_{z}$ is complicated by azimuthal phase effects (wave coupling) and the location in the model magnetosphere. The 3-D simulation data, taken close to the equatorial plane, indicate that fast mode resonance information, when examined as a function of radial distance, resides mostly in the compressional component $\left(b_{z}\right)$ of magnetic perturbations. Polarization analyses require at least two, ideally orthogonal, components of data. This may be achieved for spacecraft that carry both magnetic and electric field measurement instrumentation.

[25] The perturbation electric field data from the 3-D model are $\mathrm{e}_{\mathrm{x}}$ (radial) and $\mathrm{e}_{\mathrm{y}}$ (azimuthal) since $\mathrm{e}_{\mathrm{z}}=0$ for ideal MHD. FMR properties should be evident in the $\mathrm{e}_{\mathrm{y}}$ data, and for resonance, $e_{y}$ and $b_{z}$ are expected to show $\pm 90^{\circ}$ phase difference. This stipulates a particular value for the phase difference which might be implemented in a data analysis code by calculating either the cross phase or constructing a polarization filter using equation (2). For numerical simulations, the data are available at every grid point at each time step. This is impossible for in situ measurements in the magnetosphere. Therefore, a spacecraft was "flown" through the 3-D model near the equatorial plane at 1100 MLT to obtain perturbation electric and magnetic field time series. The cross-phase spectra obtained from the $e_{y}$ and $b_{z}$ 

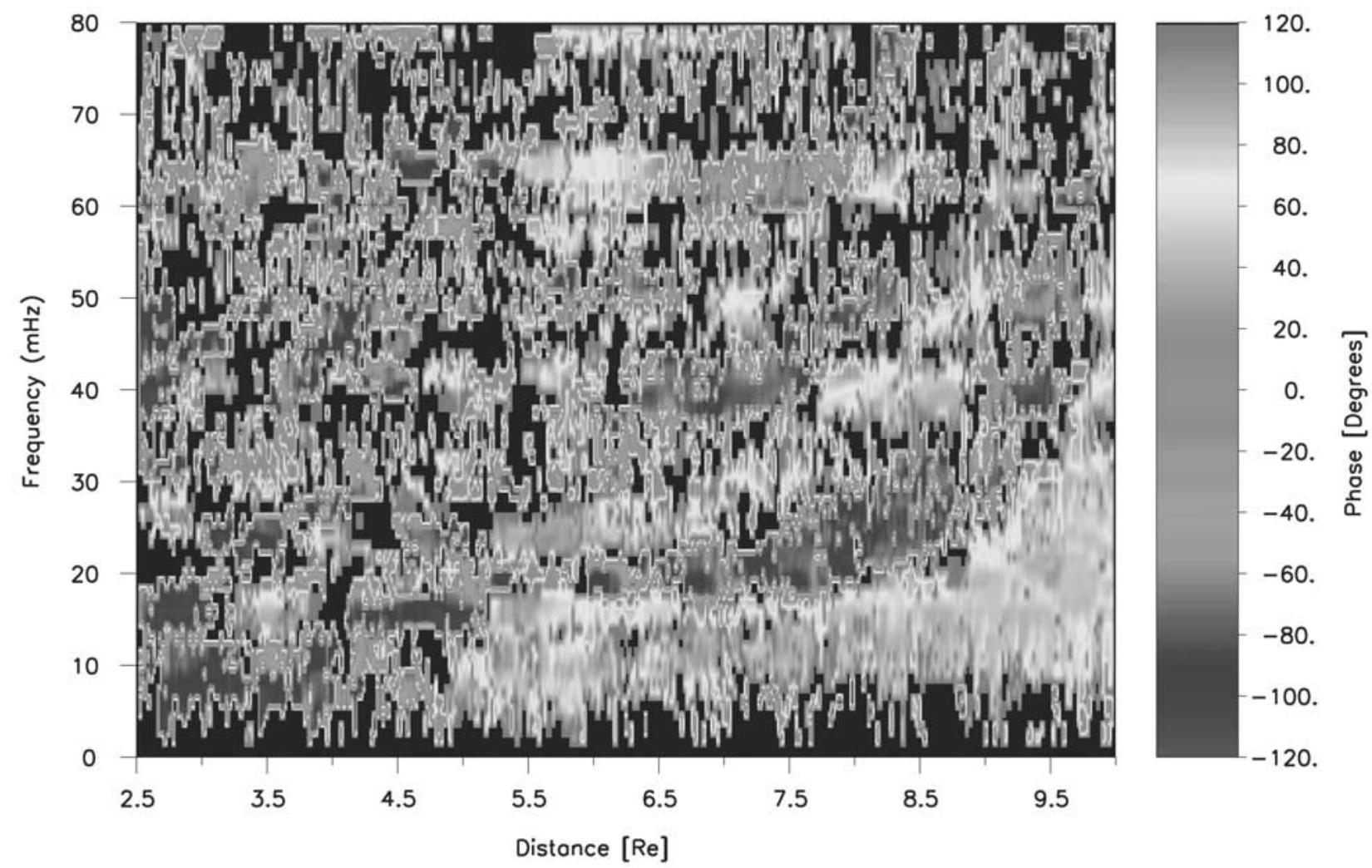

Figure 8. The cross phase of the electric field $\left(e_{y}\right)$ and magnetic field $\left(b_{z}\right)$ data from the 3-D MHD model data. At constant frequency (e.g. $39 \mathrm{mHz}$ ), the cross phase alternates with radial distance between $\pm 90^{\circ}$, as expected from of a standing wave. See color version of this figure at back of this issue.

time series are shown in Figure 8. The spacecraft "moved" at $1.3 R_{E}$ per hour (typical for AMPTE/CCE), the time series were Fourier transformed using an FFT length of $17 \mathrm{~min}$ of data followed by a shift of the FFT window by 1 min of data and so on. At a particular frequency (e.g., $39 \mathrm{mHz}$ ), Figure 8 shows the phase difference between $e_{y}$ and $b_{z}$ switches between $\pm 90^{\circ}$. This is consistent with the phase difference between the electric and magnetic fields of a standing wave obtained, for example, from electromagnetic wave reflection from a good electrical conductor. The radial distance between nodes decreases as the FMR frequency increases. Tracking some constant cross phase, (e.g., $+90^{\circ}$ ) with distance (or time), traces curves through the spectrum as seen in Figure 8.

[26] For spacecraft that record only magnetic field data, more than one spacecraft will be required to identify FMRs using cross-phase or wave polarization techniques. An examination of the $b_{z}$ data in Figure 6 suggests a detection method based on the phase variation. There is a pronounced phase shift associated with each node so that the phase difference at spatially separated locations might provide an identification signature. Alternatively, a suitable polarization vector could be chosen to detect similar amplitude and the maximum phase shift across the FMR nodes. The crossphase method was chosen since we do not know exactly what the phase shift might be, although we expect the crossphase value to depend on the resonance quality factor and the spatial separation. To test the cross-phase idea, a dual spacecraft pass through the 3-D MHD model was simulated. The simulation data were taken from grid points slightly off the equatorial plane through the 1100 MLT meridian with the second spacecraft following in the path of the leading spacecraft. The spacecraft separation was kept at $0.18 R_{E}$, twice the model radial grid spacing.

[27] Figure 9 shows the cross phase of the $b_{z}$ data from the two simulated spacecraft sampling the 3-D MHD model data. The maxima in cross phase identify regions where the phase changes more rapidly with distance which indicate nodes of the FMRs. This can be seen from a comparison with Figure 6 where the large phase changes are associated with FMR nodes. Examples in the plasma trough are the $39 \mathrm{mHz}$ FMR near 10 and $8 R_{E}$ and a $50 \mathrm{mHz}$ FMR with a node around $8.5 R_{E}$. Cross phase spectra may be used to routinely identify FLRs in ULF wave data [e.g., Waters et al., 1991] where maxima of the cross-phase track the FLR frequency. For in situ measurements, Figure 9 shows that the cross phase of radially separated $b_{z}$ data might be used to detect FMRs. When tracking FLRs using the cross phase on spacecraft data, the maxima in cross phase follows the resonant frequency, lower frequencies at larger distances from Earth. Several examples are shown in section 4. For spacecraft detection of FMRs using spatially separated $b_{z}$ data, the maxima in cross phase appear to move to higher frequencies with increasing radial distance (Figure 9) as the distance between FMR nodes decreases with increasing frequency.

\subsection{Cavity Mode Identification Criterion}

[28] From the study of MHD numerical simulation data, four methods for the detection of FMRs using spacecraft 


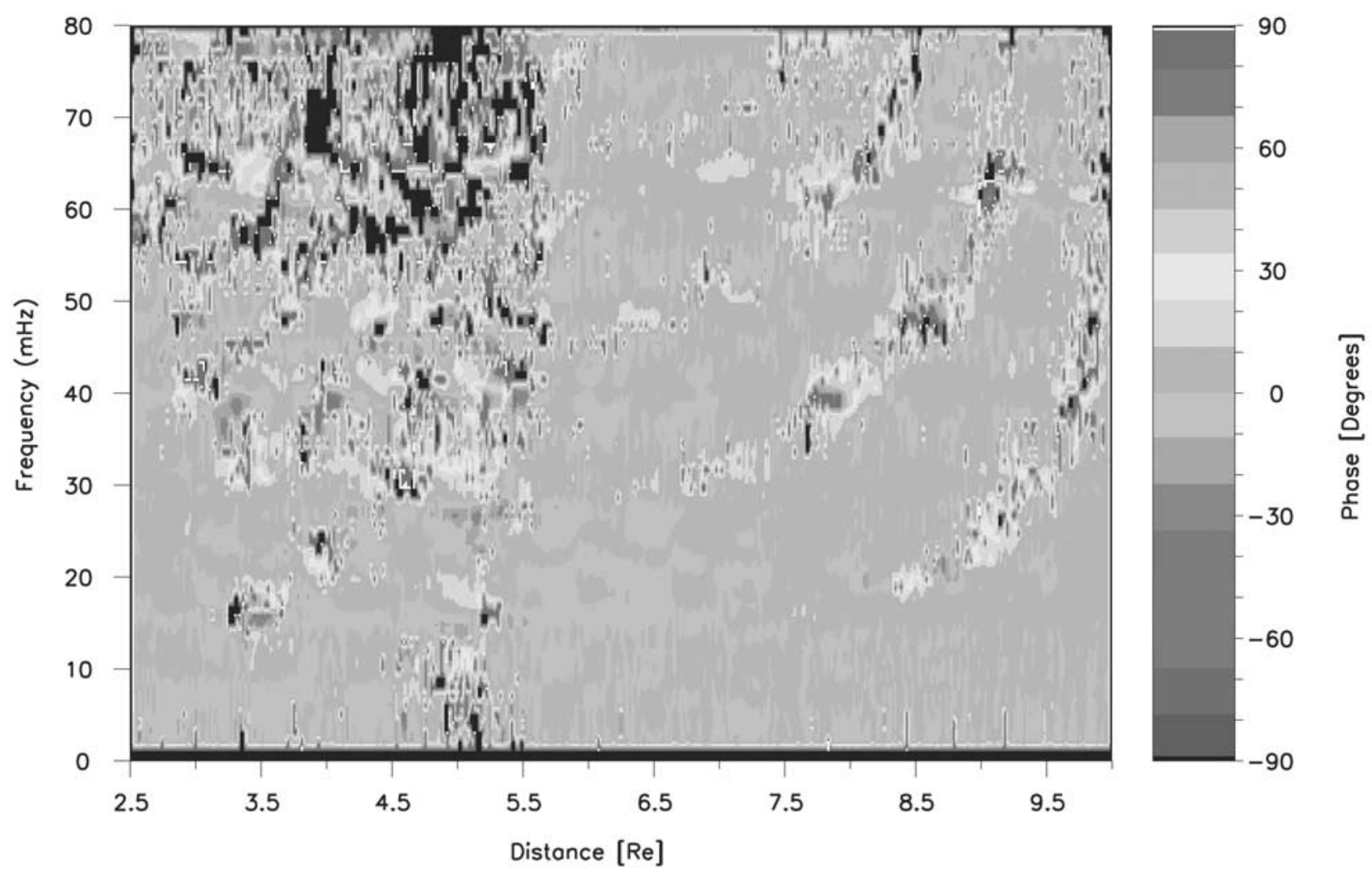

Figure 9. The cross phase of $b_{z}$ obtained from two simulated spacecraft trajectories (equatorial, radial pass and 1100 MLT) through the 3-D MHD model. This FMR detection method is based in Figure 6 where the phase is seen to change rapidly across the FMR nodes in $b_{z}$. Note for example, the large cross phase for $39 \mathrm{mHz}$ near $8 R_{E}$. The simulated spacecraft were given a trajectory speed similar to AMPTE/ CCE. See color version of this figure at back of this issue.

magnetic and electric field data have emerged. Since FMRs are expected to be predominantly in $b_{z}$, detection methods are based around this component. For single spacecraft data the methods are as follows:

1. Average the $b_{z}$ amplitude spectra obtained as the spacecraft traverses the plasma trough (e.g., Figure 5) to find any dominant peaks. The averaging process increases the signal to noise ratio.

2. Look for constant tones with radial distance in the $b_{z}$ data amplitude spectra. Ideally, node/antinode amplitude structure should be evident. These two conditions alone are insufficient to identify the signals as resonances. Standing wave structure may be deduced from analyses of both the magnetic and electric field data of the waves.

3 . Observe a $90^{\circ}$ phase difference between the compressional magnetic and azimuthal electric field data.

4. If electric field data are not available but two spacecraft are in close proximity, then for two radially separated spacecraft, examine the phase difference properties of the $b_{z}$ data. The cross-phase maxima should reveal the frequency-dependent, FMR nodal structure with radial distance (e.g., Figure 9).

\section{Satellite Data Analysis}

[29] The International Sun-Earth Explorer (ISEE) 1 and 2 spacecraft provide a configuration to search for FMRs using spatially separated measurements in addition to electric and magnetic field data from a single spacecraft. ISEE 1 and 2 magnetic field data are from identical fluxgate magnetometers described by Russell [1978]. The electric field data are from the spherical double probe experiment [Mozer et al., 1978]. The spacecraft were launched on 22 October 1977. Their elliptical orbits have apogee of $22.6 R_{E}$ and perigee around $700 \mathrm{~km}$. The data selection criteria for our study were (1) morning time trajectories as FLRs which are more likely to occur during the morning hours [Takahashi and McPherron, 1984; Anderson et al., 1990], (2) FLRs evident in the power spectra of the azimuthal magnetic component data and (3) continuous data available from both ISEE 1 and 2 for a plasma trough pass. Five intervals that have already appeared in the literature were chosen and details are provided in Table 1 . The projection of the trajectories into the equatorial plane for the five cases are shown in Figure 10. Two of the five cases are discussed below.

[30] The magnetic field data were originally provided in the GSM coordinates at 4 second resolution. We have rotated the field vectors into a mean-field-aligned (MFA) coordinate system similar to that used by Khurana and Kivelson [1989]. The instantaneous $z$ direction was defined by a 60 second running average of the data vector samples where $y$ is defined by $(\boldsymbol{z} \times \boldsymbol{r})$ for $\boldsymbol{r}$ the radial vector from the center of the Earth to the spacecraft and $\boldsymbol{x}=(\boldsymbol{y} \times \boldsymbol{z})$ completes the right-handed system. The data from both 
Table 1. Details of ISEE 1 and ISEE 2 Data Examined

\begin{tabular}{lcccc}
\hline \multicolumn{1}{c}{ Date } & Time, UT & $R_{E}$ & Separation, km & Reference \\
\hline 15 November 1977 & $0600-1145$ & $12.2-2.9$ & $140-300$ & Odera et al. $[1994]$ \\
24 November 1977 & $1800-2400$ & $12.3-5.8$ & $400-620$ & Kivelson et al. [1997] \\
11 December 1977 & $1430-1900$ & $10.5-2.2$ & $710-1450$ & Singer et al. [1979] \\
2 February 1978 & $0100-0800$ & $14.3-3.5$ & $890-2300$ & Odera et al. [1994] \\
14 February 1978 & $0000-0700$ & $14.1-3.2$ & $980-2600$ & Odera et al. [1994] \\
\hline
\end{tabular}

ISEE 1 and 2 were plotted in the time domain and visually compared. A few short duration (less than five data points) data gaps in the data from one satellite were filled using data from the other spacecraft. The data from both ISEE 1 and 2 were interpolated onto an identical time base using a cubic spline algorithm.

\subsection{November 1977}

[31] The data from ISEE 1 and 2 for 1800-2400 UT were presented by Kivelson et al. [1997]. After searching one year of ISEE 1 data, Kivelson et al. [1997, p. 1092] identified this interval as the only event that showed "persistent narrow banded ULF compressional wave power that we identify as a global mode wave." The power spectra of the $b_{y}$ and $b_{z}$ data from ISEE 1 for the inbound trajectory are shown in Figure 11. Prior to entry into the magnetosphere at $12.4 R_{E}$, wave power is quite broad band. After crossing the magnetopause at 1940 UT (1000 MLT, 12.4 $R_{E}$ ), a relatively constant tone at $24 \mathrm{mHz}$ is evident in $\mathrm{b}_{\mathrm{z}}$ which appears to satisfy FMR criteria 1 and 2 above. A number of FLR harmonics with frequency decreasing with radial distance are apparent in the $\mathrm{b}_{\mathrm{y}}$ component. Kivelson et al. [1997] reported no clear spectral peaks in the radial component data. The average of the Fourier transform, amplitude spectra of the magnetic perturbation data recorded when ISEE 1 was inside the magnetosphere are shown in Figure 12. The ratio of the $b_{x}$ to $b_{z}$ amplitude is similar to the 3-D MHD simulation data. Furthermore, the peaks in the $b_{\mathrm{y}}$ average spectra suggest FLR activity similar to Figure 5 around $25 \mathrm{mHz}$. This event was also observed over a large spatial extent, detected by GOES 2, located in the afternoon sector and also by SMS 2 located in the morning sector [Kivelson et al., 1997].

[32] These properties strongly suggest that the $24 \mathrm{mHz}$ signal was a global mode. The question is whether this event shows resonance properties. The event satisfies criteria 1 and 2 above as they are tests involving amplitude properties. Tests for resonance characteristics should also be devised and applied. The cross-phase spectra between ISEE 1 and 2 data for $b_{y}$ and $b_{z}$ are shown in Figure 13. The $b_{y}$ cross-phase spectra show multiharmonic FLRs, increasing in frequency as the two spacecraft move toward the Earth and FLR excitation appears for frequencies < $\sim 40 \mathrm{mHz}$ after entry into the magnetosphere. The crossphase spectra of the $b_{z}$ data do not resemble the 3-D MHD data in Figure 9. There are no clear maxima in cross phase that would identify FMR nodes and the cross phase is consistently quite close to zero at $24 \mathrm{mHz}$ for radial distances less than $12 R_{E}$. However, this particular method depends on the spacecraft separation and most probably the resonance quality factor as this alters the variation of phase with radial distance. The 3-D model cross-phase data (Figure 9) were computed for a spacecraft separation of $1150 \mathrm{~km}$ with maxima values of $\sim 40^{\circ}$ obtained. For the
24 November, ISEE 1 and 2 were separated by $400 \mathrm{~km}$ at $12 R_{E}$ widening to $620 \mathrm{~km}$ at $6 R_{E}$. Given the smaller separation and the relatively low resonance $Q$ estimated by Kivelson et al. [1997], it may be argued that any resonance signature obtained from cross-phase maxima is reduced below detection.

[33] An alternative test for resonance properties is available using the electric field data from ISEE 1. The electric and magnetic field perturbation data from ISEE 1 for 24 November 1977 were analyzed by Chi and Russell [1998]. Their Figure 1 shows the time series with waves of the same frequency in the electric and magnetic data, the average frequency over 2050-2130 UT being $24 \mathrm{mHz}$. The resonant condition in point 3 above was tested by Chi and Russell [1998], and the results can be seen in their Figures 5 and 10. In comparison with a clear resonance event at 4 $\mathrm{mHz}$ for ISEE 1 data recorded on 3 November, 1977, the 24 $\mathrm{mHz}$ signal on 24 November 1977 showed a phase difference between the wave electric and magnetic field data that was ' a strong function of time ...' which'... suggests that the spacecraft was not observing a standing wave structure" [Chi and Russell, 1998, p. 29,486].

\subsection{February 1978}

[34] A representative example of the remaining four events examined is the data obtained by ISEE 1 and 2 on 14 February 1978. These data were also discussed by Odera et al. [1994]. Power and phase spectra of the magnetometer data for the inbound pass are shown here in Figures 14 and 15,

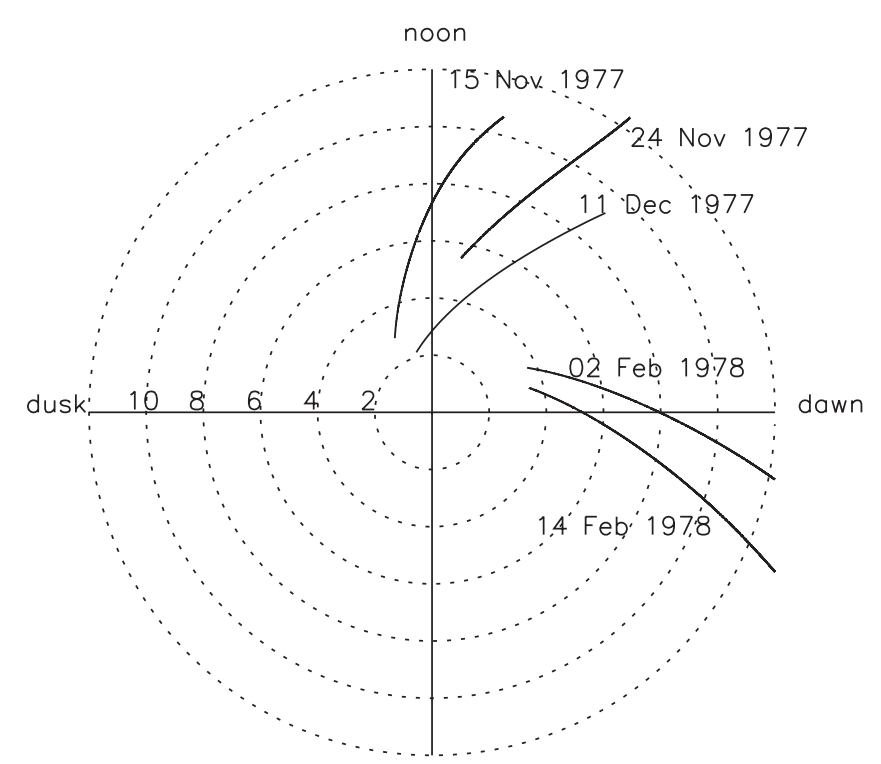

Figure 10. Projection of the ISEE 1 and 2 trajectories onto the equatorial plane for the data listed in Table 1. 


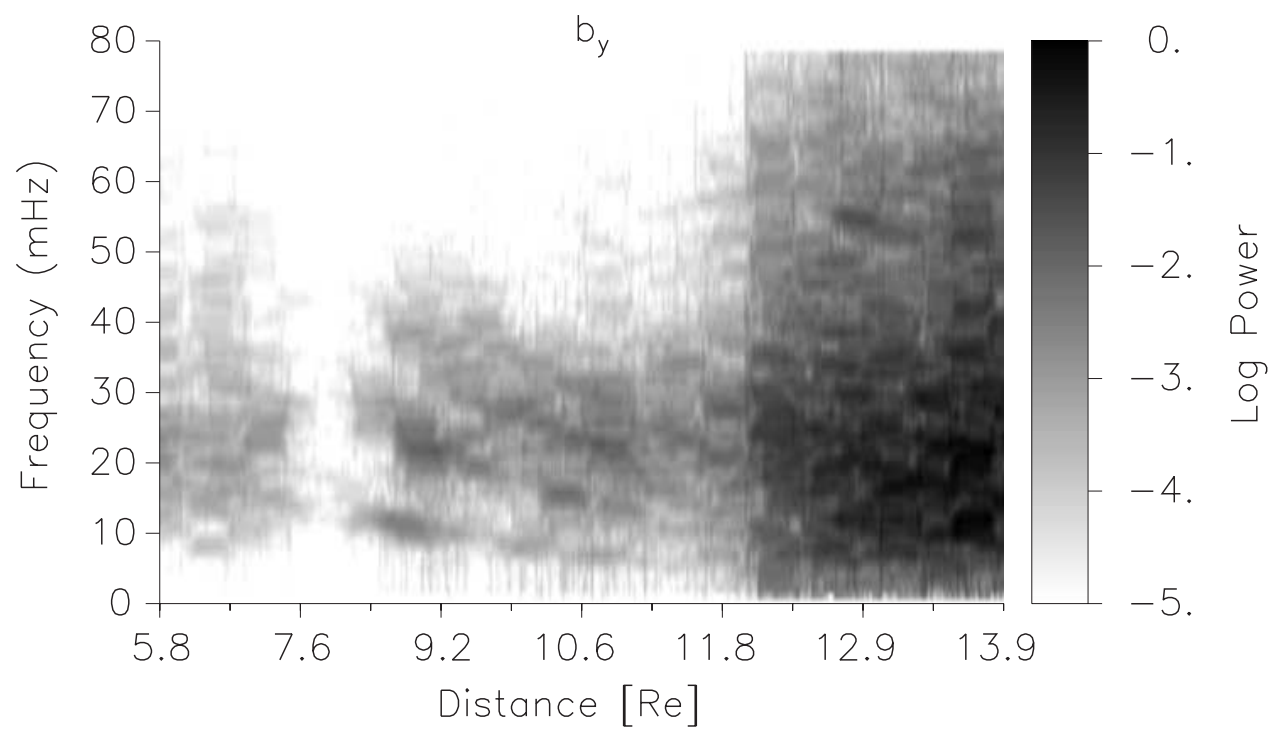

(a)

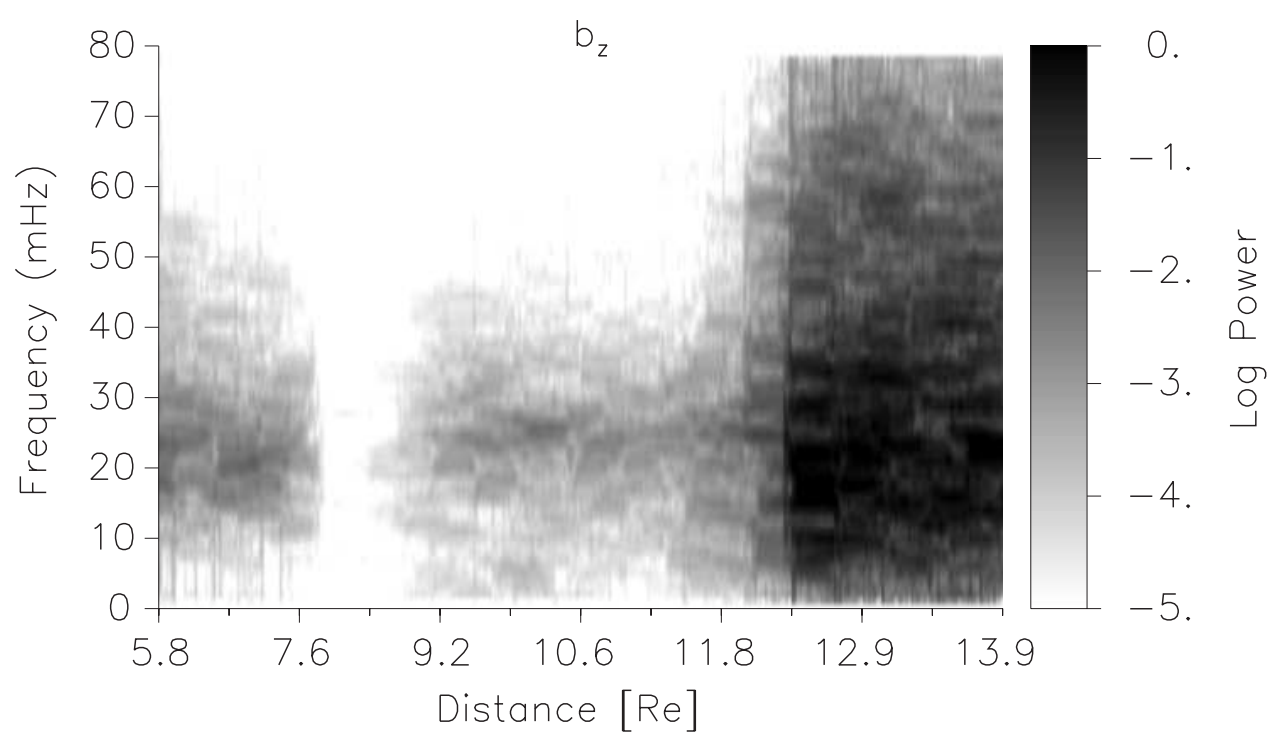

(b)

Figure 11. Power spectra of ISEE 1 data for $0200-0730$ UT, 14 February 1978. (a) The azimuthal $\left(b_{\mathrm{y}}\right)$ channel and (b) the field-aligned $\left(\mathrm{b}_{\mathrm{z}}\right)$ data. ISEE 1 was inbound and crossed the magnetopause at $12.4 R_{E}$.

respectively. The spacecraft separation varied from 980 to $2600 \mathrm{~km}$ (Table 1). The power spectra of the azimuthal component data (Figure 14a) show FLRs increasing in frequency up to $\sim 40 \mathrm{mHz}$ as the spacecraft move earthward. The cross phase between the ISEE 1 and $2 b_{y}$ data are not as clear as Figure 13. FLRs can be quite narrow in radial extent [Singer et al., 1979, 1982], and the spatial separation of the spacecraft might be too large for the cross phase to provide a definitive signature, or the resonance quality factor may be smaller for these data. For FMRs, the distance over which the perturbations are coherent is expected to be of the order of a few $R_{E}$. If the signals are coherent, the cross-phase spectra should indicate consistent values at those frequencies. Figure $15 \mathrm{~b}$ shows consistent cross phase in two regions of the spectra. For the very low frequencies
(3-10 $\mathrm{mHz}$ ), the signals appear coherent over the spacecraft separation distance into $\sim 8 R_{E}$. The $20-50 \mathrm{mHz}$ band signals are coherent over the spacecraft separation distance between $\sim 6.5$ to $4 R_{E}$. Therefore, for radial distances greater than $\sim 6.5 R_{E}$, the $20-50 \mathrm{mHz}$ band appears to have a spatial coherence $<2,600 \mathrm{~km}$ or $0.4 R_{E}$, much smaller than the few $R_{E}$ expected for FMRs.

\section{Discussion}

[35] The excitation of FLRs in the Earth's magnetosphere is thought to originate in a wave generation region around the magnetopause where radially agile fast mode waves transports energy to resonant shells in the magnetosphere forming FLRs. Despite the numerous observations of FLRs 

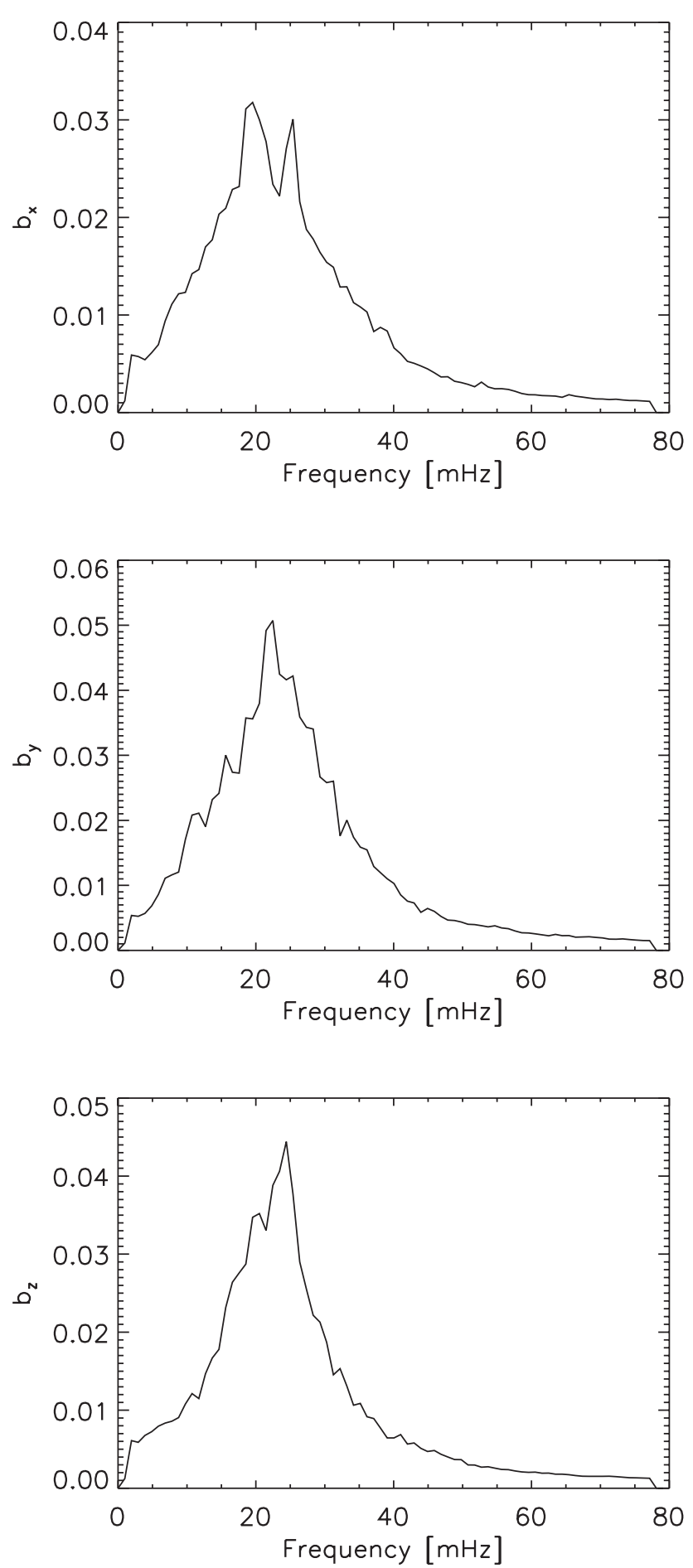

Figure 12. The average of the Fourier transform, amplitude spectra (in nT) of ISEE 1 data for 2000-2400 UT, 24 November 1977. This excludes the magnetosheath data from 12.4 to $13.9 R_{E}$ in Figure 11.

in spacecraft data, the experimental identification of FMRs in the plasma trough remains illusive. Based on 1-, 2- and 3-D numerical models, several descriptions of FMRs have emerged including cavity and waveguide modes and more recently, virtual resonances. From the lack of experimental evidence, it appears that cavity modes, as described by
1-D MHD models, do not exist in the plasma trough region of the magnetosphere. However, both numerical modeling and experimental evidence supports the existence of FMRs in the form of virtual resonances within the plasmasphere. There is ample experimental evidence for FLRs in the plasma trough region, and if the ideas of FLR excitation through fast mode coupling are to be retained then waveguide modes and/or non-FMR fast mode waves appear to be the remaining options. The persistent observation of the more broadband type compressional energy from the AMPTE/CCE data suggests the latter option. However, both Rickard and Wright [1995] and Allan et al. [1997] demonstrate that spacecraft detection of waveguide modes may not show monochromatic structure but would appear more like the observed AMPTE/CCE spectra. These studies used 2-D MHD models. Allowing wave leakage at the outer boundary in the 3-D MHD model of Lee and Lysak [1999] allows some insight into this effect by examining the simulation data away from noon.

[36] The data from the 3-D MHD model presented so far were taken from a line of grid points close to the equatorial plane at 1100 MLT. The data were taken after the initial impulse had died away and more steady state conditions prevailed. Based on Figure 6 of Rickard and Wright [1995], including time intervals when the energization pulse was active would alter the amplitude of fast mode waves and FLRs in the near vicinity of the pulse which a spacecraft is likely to miss. Therefore it seems more pertinent to concentrate on the more global steady state oscillations keeping in mind that local variations in amplitude may occasionally occur if the spacecraft happens to sample data from regions near the excitation source in the real magnetosphere. Before we discuss data from the dawnside, where more waveguidelike energy is likely to be found, a few comments on the 3-D model data from off equatorial, radial trajectories near 1200 MLT are in order. FLRs in the 3-D model data such as those in Figures 3 and 4 of Lee and Lysak [1999] are seen in the $b_{y}$ (azimuthal) component when taken from radial passes at all MLTs and latitudes of the model. For an off-equatorial line at 1200 MLT, the $b_{z}$ data from the 3-D model were analyzed for a radial pass starting at a sunward distance of $8.9 R_{E}$ and northward distance off the equatorial plane of $2.8 R_{E}$. The cross phase of these data was very similar to those from the equatorial plan, shown in Figure 9. Therefore, on the basis of the 3-D model data, FMR spatial structure and their detection in spacecraft data is relatively insensitive to the proximity of the spacecraft trajectory to the equatorial plane.

[37] The data recorded on 14 February 1977 by ISEE 1 and 2 were for an inbound pass near dawn. In the 3-D MHD model, the magnetopause boundary increasingly leaks wave energy as the location moves away from noon. The results of FMR amplitude tests on the 3-D model data taken along a line at 0600 MLT and the equatorial plane are shown in Figures 16 and 17 and may be compared with the 1200 MLT data in Figures 5 and 6 . The $b_{\mathrm{y}}$ component at $39 \mathrm{mHz}$ in Figure 16 shows little enhancement from coupling to $b_{z}$. The radial structure of the $39 \mathrm{mHz}$ signal (identified from Figures 5 and 6) is shown in Figure 17. The $b_{z}$ component shows a plasmasphere virtual resonance, but the plasma trough signal is very small in comparison. The cross phase of the two simulated spacecraft, $b_{z}$ data (not shown) revealed no resonance signatures in the plasma trough as 


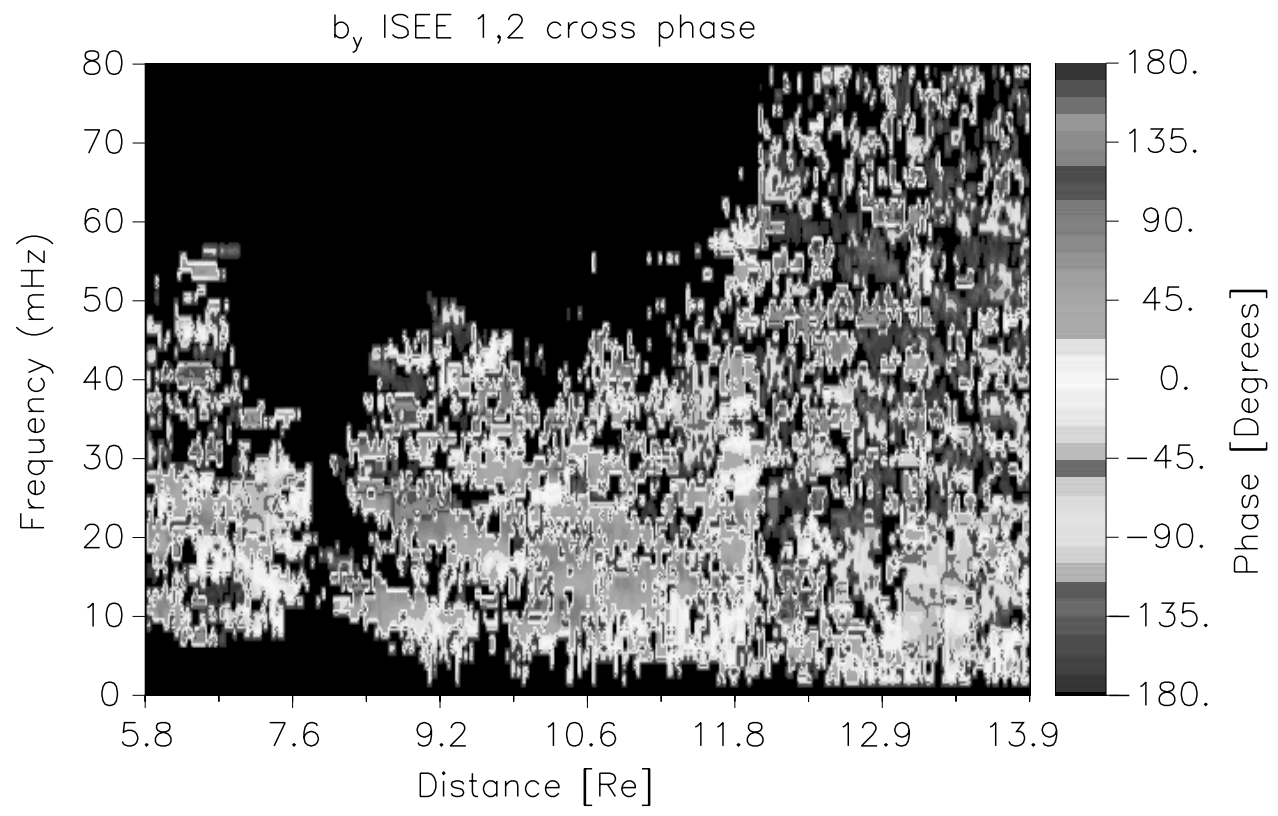

(a)

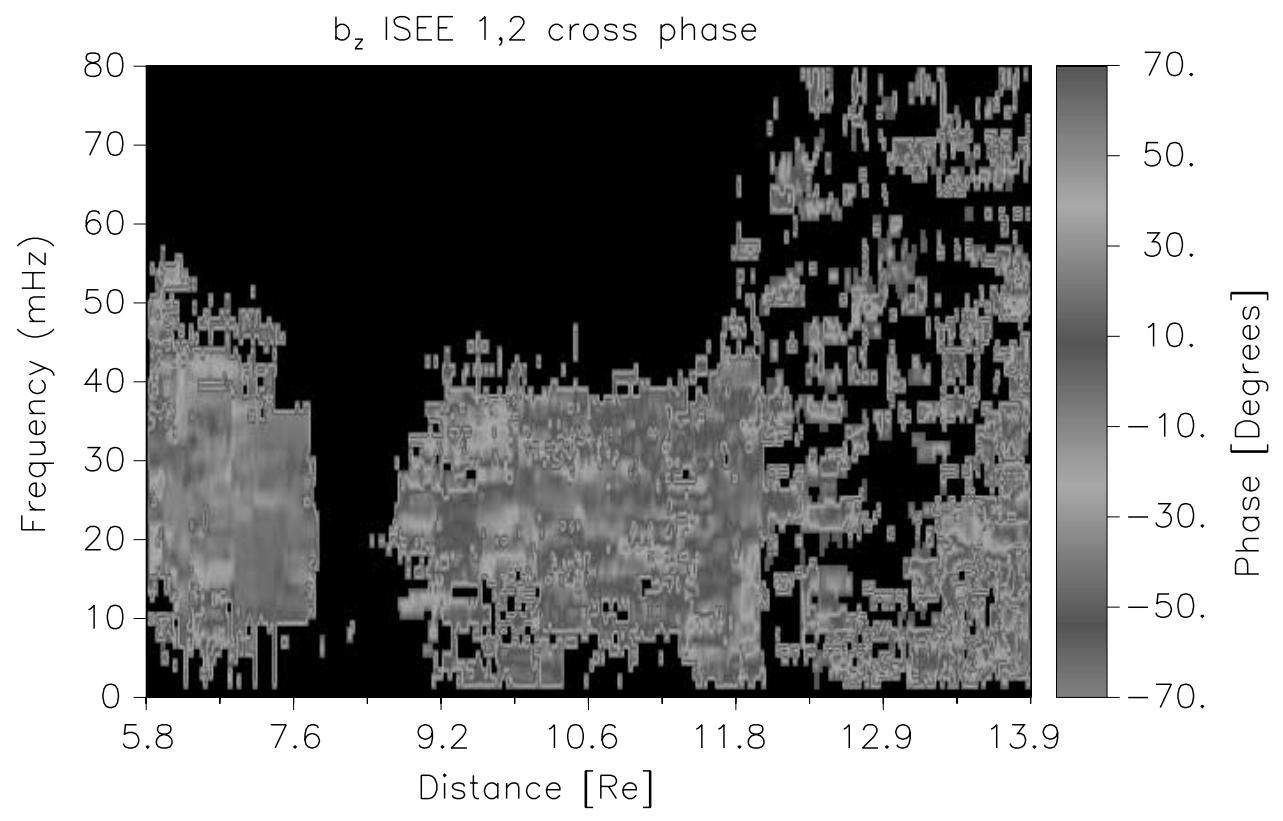

(b)

Figure 13. The cross phase spectra of ISEE 1 and 2 data for 1800-2400 UT, 24 November 1977. (a) The $b_{y}$ components showing multiharmonic FLRs. (b) The $b_{z}$ component data with a change in scale of the phase to highlight the smaller cross phase. The spacecraft entered the magnetosphere at $12.4 R_{E}$. See color version of this figure at back of this issue.

seen, for example, in Figure 9. The analysis for $90^{\circ}$ phase difference between $b_{z}$ and $e_{y}$ showed that for most of the plasma trough region, the phase difference at $39 \mathrm{mHz}$ was within $\pm 20^{\circ}$. The FMR property of $\pm 90^{\circ}$ phase difference between $b_{z}$ and $e_{y}$ reappeared in the plasmasphere. The persistence of FLR energy in the 3-D model plasma trough region may be due to the perfect reflection boundary condition at the ionosphere. If ionosphere dissipation were included, the results of Lee et al. [2000] indicate that the properties of FMRs would remain unchanged and for reasonable conductivities, the FLRs persist for at least 10 min after the excitation pulse has ceased.

[38] Spacecraft data readily show FLRs in the 10 to 80 $\mathrm{mHz}$ frequency range. The 3-D model data show that FMRs near 1200 MLT have relatively larger amplitudes in this frequency range and are weaker for $<10 \mathrm{mHz}$, especially around the 1.3, 1.9, 2.3, 2.6 ... mHz region. An estimate of the frequencies of FMRs based on twice the travel time 


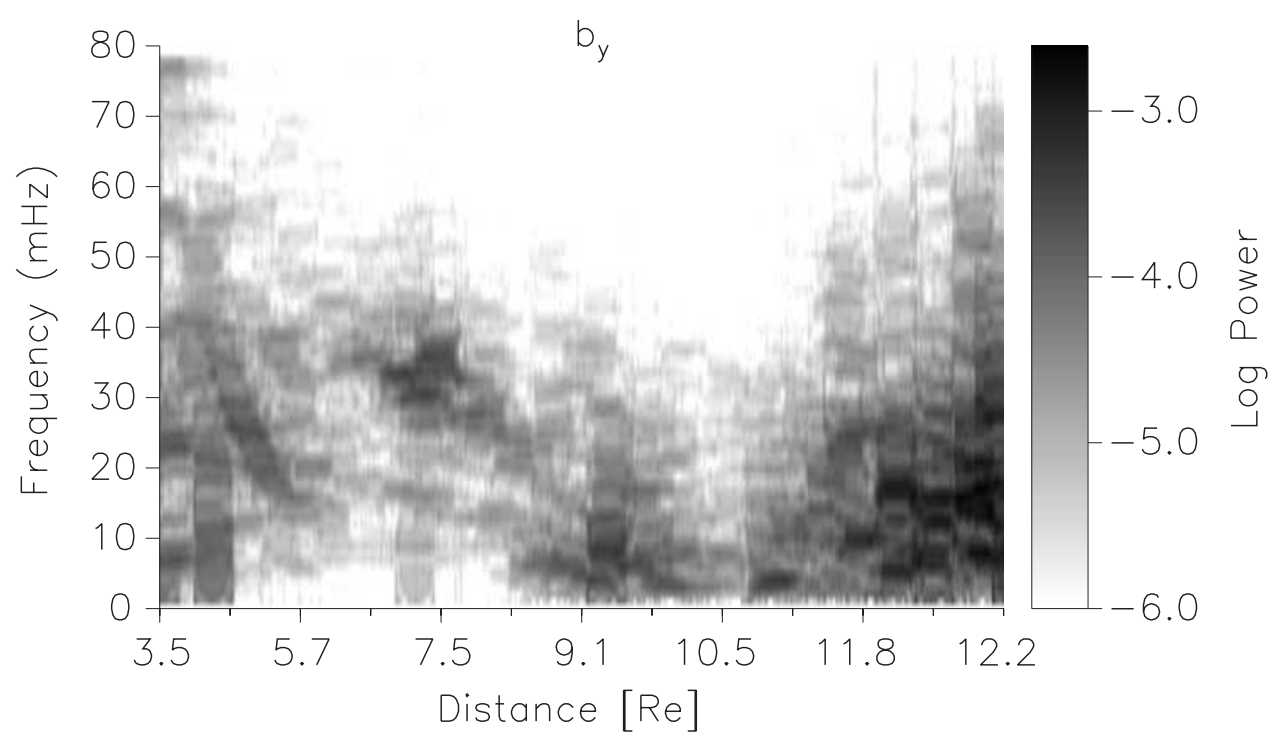

(a)

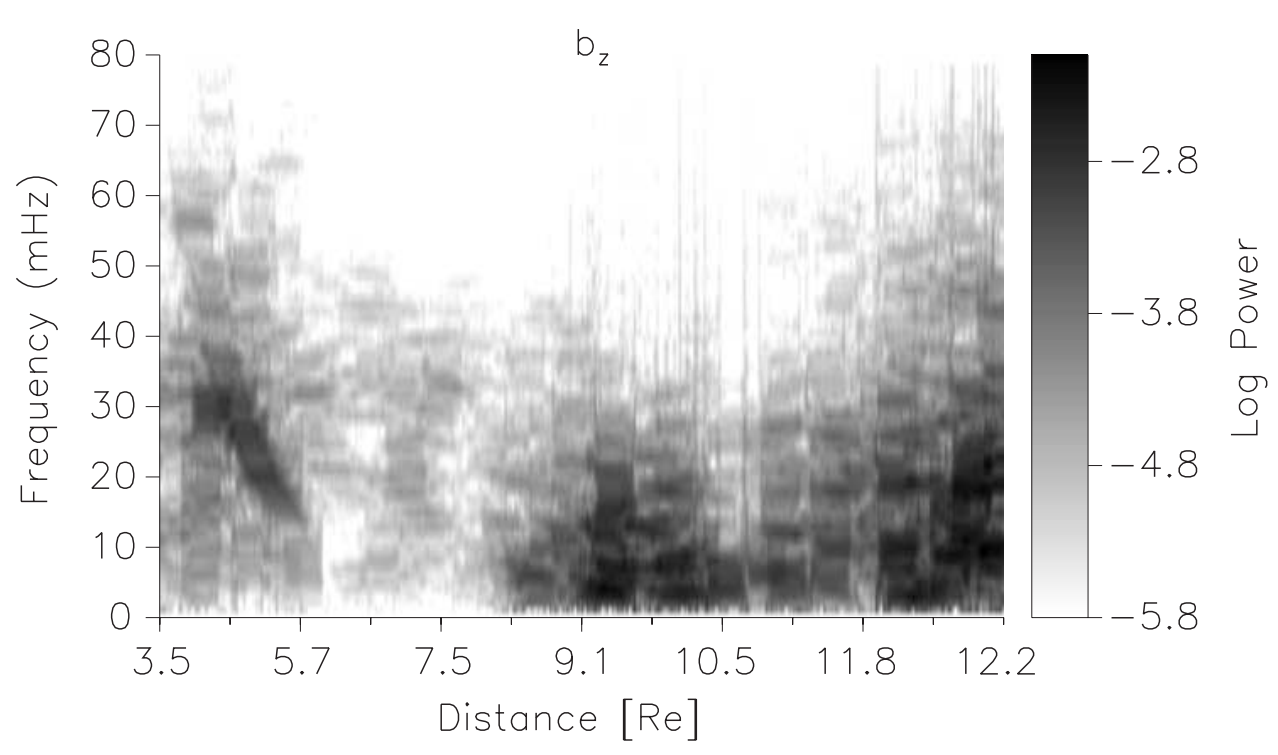

(b)

Figure 14. Power spectra of data from ISEE 1 for 0200-0730 UT, 14 February 1978: (a) azimuthal $\left(b_{y}\right)$ data and $(b)$ field-aligned $\left(b_{z}\right)$ data.

between the ionosphere and the magnetopause excludes FMRs with such low frequencies, unless quarter wave resonances occur. Studies of quarter wave modes in a uniform magnetosphere by Mann et al. [1999] neglected the role of the plasmasphere. The magnetosphere can present two coupled cavities to ULF waves. This is more important for low frequencies where the radial wavelengths are large. The 3-D MHD data include wave energy leakage at the magnetopause and shows that FMRs disappear in the plasma trough region but can remain active inside the plasmasphere.

[39] The 3-D model data were taken from steady state conditions. Due to variations in the solar wind velocity and density, the real magnetosphere is expected to be subjected to more impulsive activity. Furthermore, realistic ionosphere conductivities create damping for FLRs. Therefore, some external energy source is required to produce FLRs that persist for many hours. The excitation of FLRs by more impulsive type wave sources has been examined theoretically in studies such as Chen and Hasegawa [1974b] and Allan et al. [1986a, 1986b]. Experimental estimates of decay times of transient ULF pulsations observed by ground based instrumentation range from $125 \mathrm{~s}$ [Glassmeier et al., 1984] to $1000 \mathrm{~s}$ [Allan et al., 1985]. By comparing the frequency of toroidal resonances measured by AMPTE/ CCE, Anderson et al. [1989] obtained an effective FLR ringing time of $1000 \pm 300 \mathrm{~s}$ in the plasma trough. It appears that FLRs in the plasma trough have quite long 


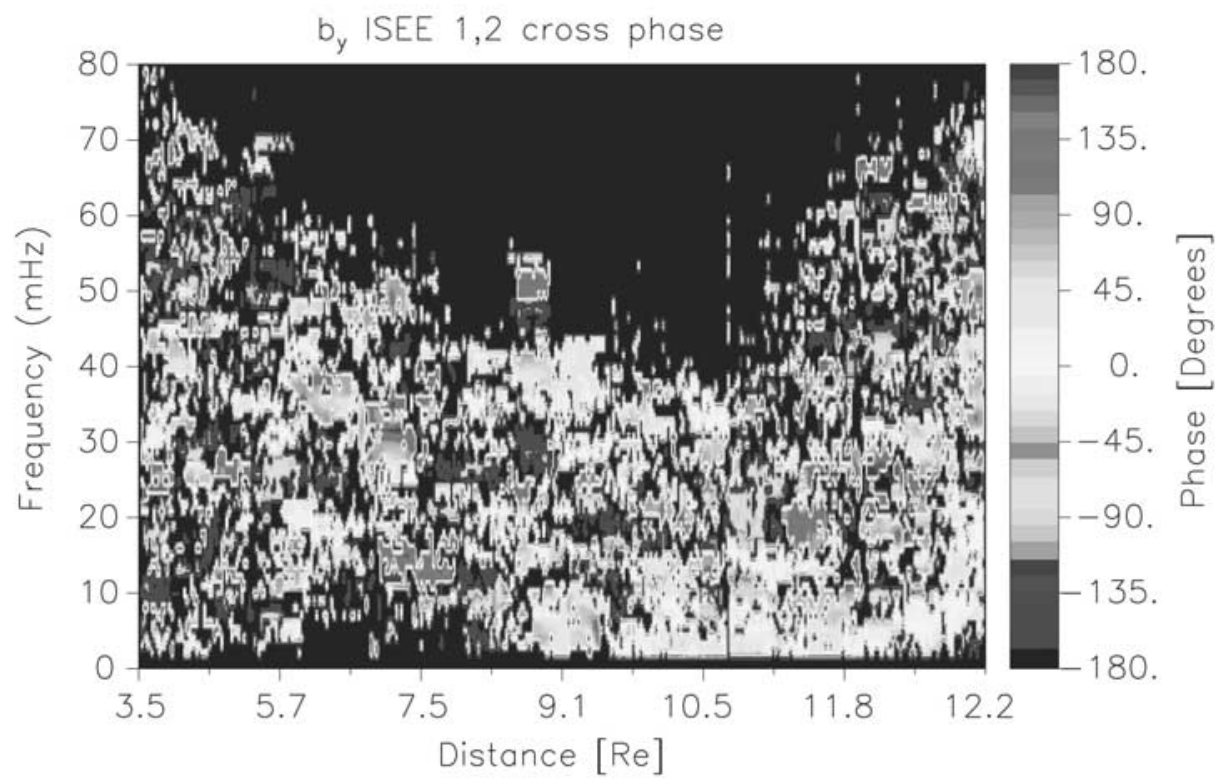

(a)

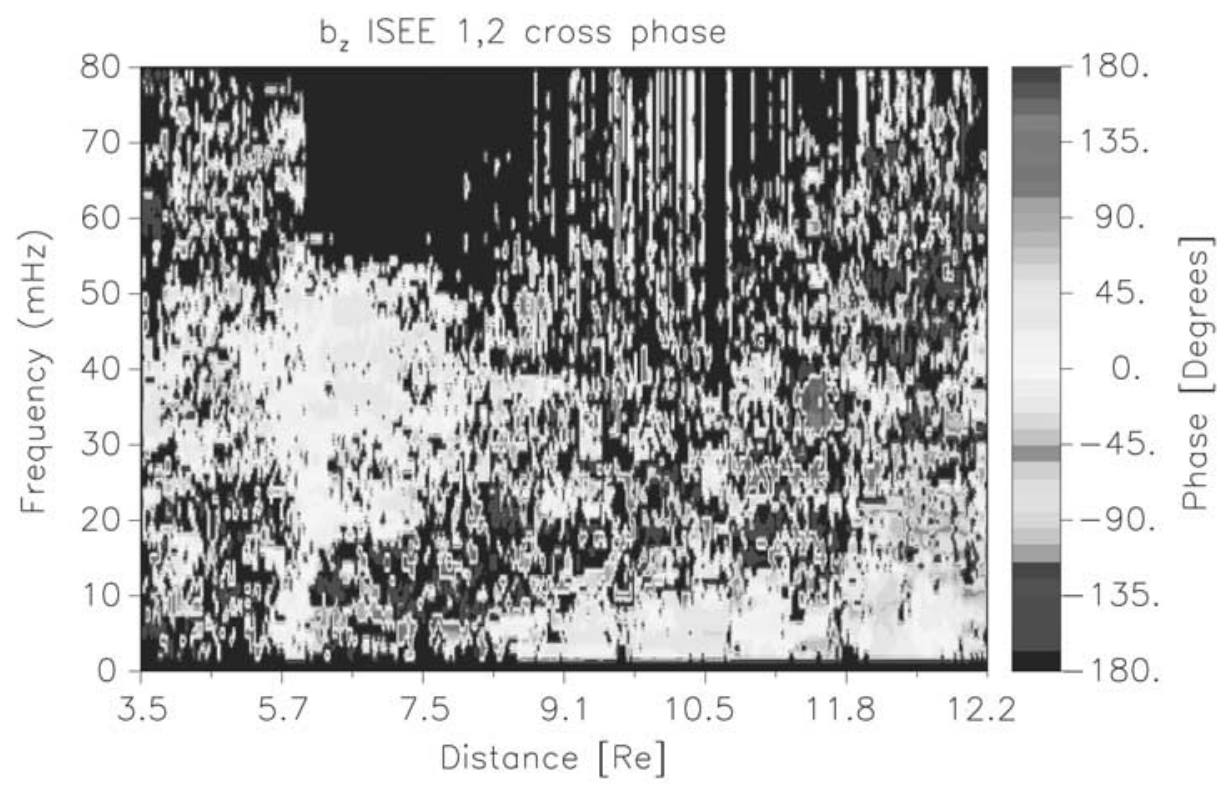

(b)

Figure 15. Cross phase of data from ISEE 1 and 2 for 0200-0730 UT, 14 February 1978: (a) cross phase of ISEE 1 and 2, azimuthal $\left(b_{y}\right)$ data and (b) cross phase of ISEE 1 and 2, $b_{z}$ data. Note the lack of consistent, $\mathrm{b}_{\mathrm{z}}$ cross-phase values for frequencies greater than $10 \mathrm{mHz}$ and for distances greater than $8 R_{E}$. See color version of this figure at back of this issue.

decay times and that small field perturbations are sufficient to generate multiharmonic FLRs. In contrast, the 3-D model data show that wave leakage at the outer boundary is sufficient to over damp FMRs. The 3-D model was initiated by a nightside, $\sim 1$ min duration pulse that produced multiharmonic FLRs throughout the whole simulated magnetosphere. Most previous studies have focused on the dayside region as the origin of energy sources for FLRs. This is due to the observed correlations of Pc3-4 wave amplitude and frequency with solar wind and interplanetary magnetic field characteristics. The 3-D model data suggest that nightside perturbations or those associated with tail dynamics may also contribute to FLR excitation, perhaps even the 1.3, 1.6, $1.9 \ldots \mathrm{mHz}$ frequencies, which appear to be too low in frequency to be driven by dayside FMRs. It would be informative to run the 3-D MHD simulation using repetitive impulse excitation. However, due to computing constraints this is not possible at this time.

[40] A number of possible reasons for the failure to find FMRs in spacecraft data are given by Kivelson et al. [1997]. 

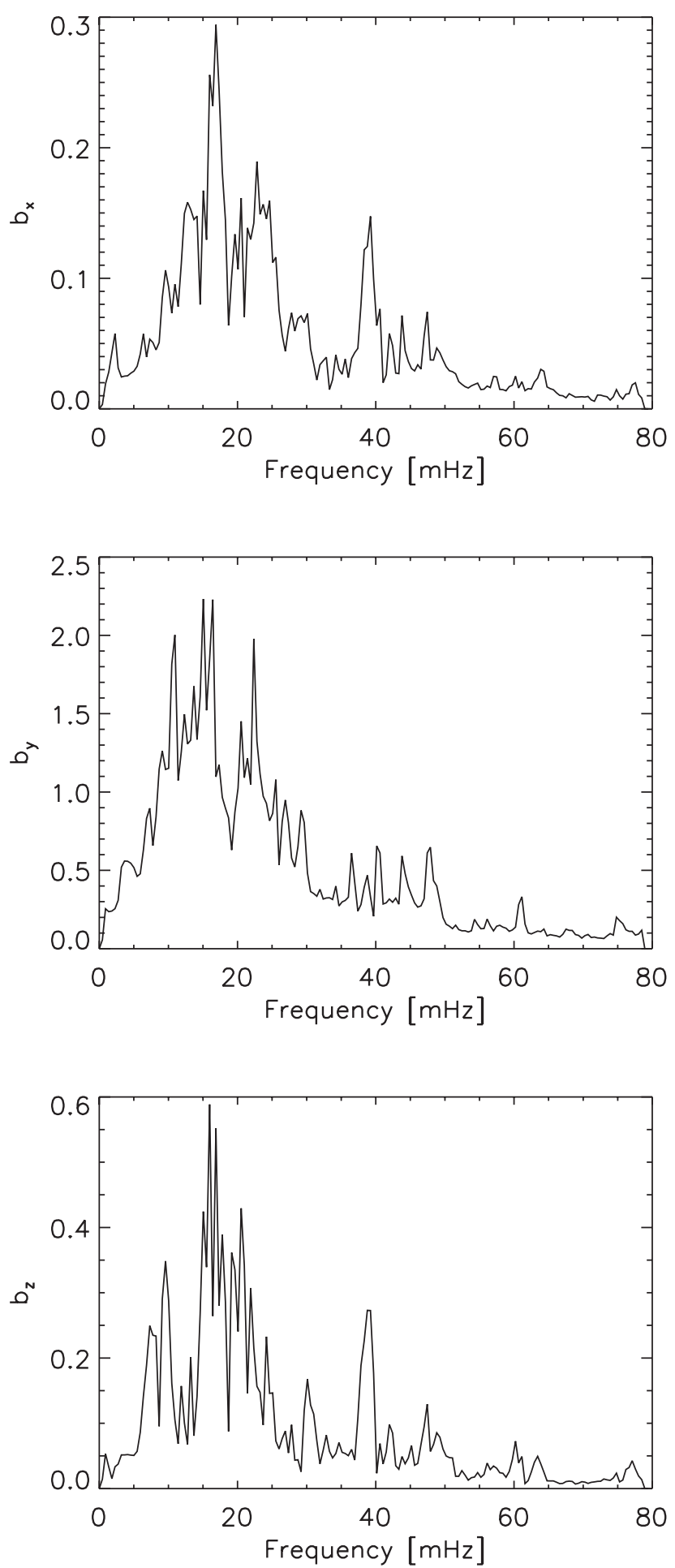

Figure 16. Average of the Fourier transform, amplitude spectra of data from the 3-D MHD model. The data were sampled from a radial line of grid points in the equatorial plane at 0600 MLT.

Most of these highlight the extraordinary magnetospheric conditions that must prevail (resonance $Q, \mathrm{IMF}$ cone angle, stable magnetosphere cavity) for spacecraft to adequately sample such a ULF wave structure. One point concerns the probable signal levels and special data processing that may need to be used. The 3-D model data from the dayside, where FMRs couple to FLRs, show that the amplitudes of $b_{y}$ and $b_{z}$ are comparable (Figures 5 and 6). This suggests that if the amplitude resolution of a spacecraft magnetometer is sufficient to detect FLRs, then the amplitude of FMRs should also be sufficiently above the noise level to facilitate detection. Extra data processing in the form of cross-phase or polarization analyses has been suggested as a method for determining if data on the $b_{z}$ component have resonance characteristics. A difficulty with FMR detection is the interplay between the expected spatial size of the wave structure, the spacecraft magnetometer sample rate and the speed of the spacecraft through the structure. These factors make it difficult to detect FMRs using data from spacecraft in the plasmasphere. In the plasma trough, the cross phase of data from two spatially separated spacecraft
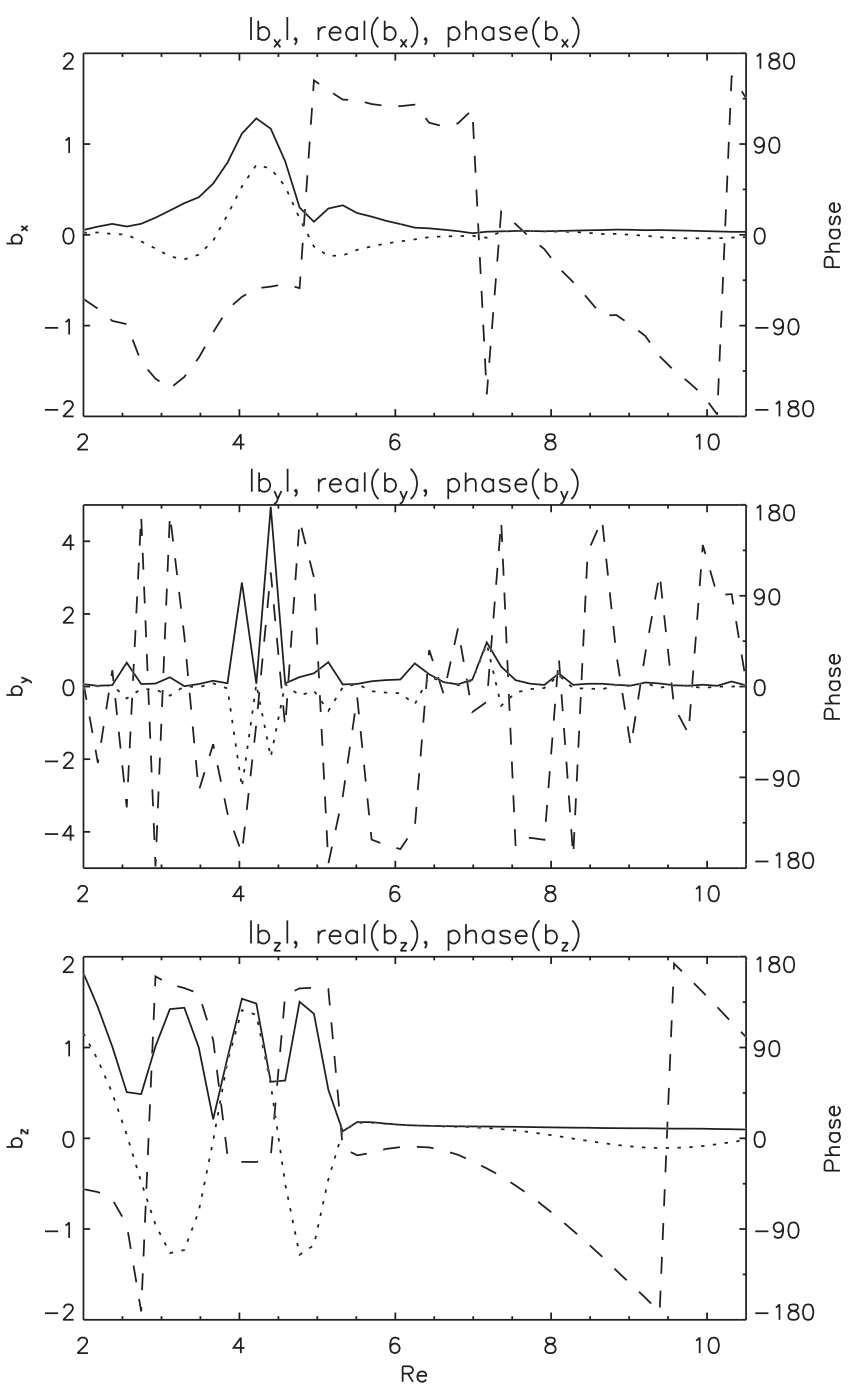

Figure 17. The dawnside magnetic field data from the 3-D MHD model. The absolute value (solid), real part (dotted) and phase (dashed) of the radial $\left(b_{x}\right)$, azimuthal $\left(b_{y}\right)$ and field-aligned $\left(b_{z}\right)$ at $39 \mathrm{mHz}$ are shown. The data were sampled from a radial line of grid points in the equatorial plane at 0600 MLT. The $b_{z}$ signature of an FMR in the plasma trough has disappeared. 
is sensitive to their spatial separation and the resonance quality factor, $Q$, of the FMR. However, FMRs and propagating fast mode energy, discussed in the context of FLR excitation, is usually considered to have coherence over spatial separations of at least a few $R_{E}$. Based on the data for 14 February 1978 the radial distance for coherence of the $b_{z}$ signal is smaller than $0.4 R_{E}$. These conditions were still sufficient for FLRs to exist throughout the plasma trough (Figures 14 and 15). A consistent result from the ISEE and AMPTE/CCE spacecraft data is that FLRs may be quite prominent in the $b_{y}$ (azimuthal) data even when the $b_{z}$ data have virtually zero amplitude.

\section{Conclusions}

[41] The excitation of FLRs in the Earth's magnetosphere by resonant fast mode energy has been the favored explanation for over 20 years. A number of studies have suggested identification of FMRs in spacecraft data. However, these were based on expected wave amplitude characteristics that do not consider the phase structure of resonance. In this paper we have provided a set of criteria that may be used to test for resonance of fast mode waves using spacecraft data. The amplitude characteristics used in previous studies have been included, but these are extended to include tests for resonance properties using the signal phase information.

[42] To facilitate detection, the expected structure of FMRs has been examined using both 1-D and 3-D MHD simulation data. The 3-D data show that FMRs should have comparable amplitude as the often detected FLRs. The formation of FMRs is more likely to occur on the dayside where wave reflection properties at the outer boundary are more favorable for the formation of radial resonance structures. The FMR detection criteria were tested on the 3-D simulation data and identified FMRs for a noon, radial pass through the simulated magnetosphere. The data from six radial passes of AMPTE/CCE, ISEE 1 and 2 were analyzed using the proposed tests for FMRs. The analysis of the spacecraft data failed to identify FMRs in the plasma trough region. In fact, the radial distance over which the $b_{z}$ signal showed a consistent phase relationship was less than $0.4 R_{E}$, much too small for FMRs that are expected to extend for several $R_{E}$. There have been a number of events reported (e.g., Figures 11 and 13) that appear to be FMRs based on the first two, amplitude-based criteria. However, these data failed the subsequent resonance property tests based on the phase relationship between the magnetic and electric field perturbations.

[43] The analysis in this paper does not preclude FLR excitation by impulse initiated waveguide modes. These have been studied theoretically in 2-D geometries [Rickard and Wright, 1995; Mills et al., 1999]. However, these modes may prove difficult to detect using spacecraft as they tend to propagate close to and parallel with the magnetopause. Future work, depending on computing resources, would focus on impulsive sources and waveguide modes in a 3$\mathrm{D}$, open magnetosphere geometry including realistic ionospheric conductivities. It may turn out that FMRs are not required to persist in order to excite multiharmonic FLRs. Since FLRs appear to have long decay times $(\sim 1000 \mathrm{~s})$ while FMRs are more likely to be over damped, any impulsive type activity in the magnetosphere might contrib- ute to FLR excitation. The 3-D MHD simulation data used in this paper were initiated by $\mathrm{a} \sim 1 \mathrm{~min}$ pulse on the nightside. However, FLR signals are quite often seen to last for hours and extend from the magnetopause into the inner plasmasphere which means that the average repetition rate of any impulsive source must be less than the $1000 \mathrm{~s}$ decay time of FLRs and be capable of large spatial-scale excitation. Such an energy source has not yet been identified.

[44] Acknowledgments. The authors thank G. Le, P. J. Chi, and C. T. Russell for assistance with the ISEE spacecraft magnetometer data. Work at JHU/APL was supported by the NSF grant ATM-9901102 and the NASA grant NAG5-8964.

[45] Janet G. Luhmann thanks Richard E. Denton and another referee for their assistance in evaluating this paper.

\section{References}

Alfvén, H., C. G. Fälthammar, Cosmical Electrodynamics, Oxford Univ. Press, New York, 1963.

Allan, W., S. P. White, and E. M. Poulter, Magnetospheric coupling of hydromagnetic waves: Initial results, Geophys. Res. Lett., 12, 287 , 1985.

Allan, W., S. P. White, and E. M. Poulter, Impulse-excited hydromagnetic cavity and field-line resonances in the magnetosphere, Planet. Space Sci., 34, 371, 1986a.

Allan, W., E. M. Poulter, and S. P. White, Hydromagnetic wave coupling in the magnetosphere: Plasmapause effects on impulse-excited resonances, Planet. Space Sci., 34, 1189, 1986 b.

Allan, W., F. W. Menk, B. J. Fraser, Y. Li, and S. P. White, Are low-latitude Pi2 pulsations cavity/waveguide modes?, Geophys. Res. Lett., 23, 765, 1996.

Allan, W., A. N. Wright, and D. R. McDiarmid, Spacecraft transits across simulated field line resonance regions, J. Geophys. Res., 102, 14,407, 1997.

Anderson, B. J., An overview of spacecraft observations of $10 \mathrm{~s}$ to $600 \mathrm{~s}$ period magnetic pulsations in the Earth's magnetosphere, in Solar Wind Sources of Magnetospheric Ultra-Low Frequency Waves, Geophys. Monogr. Ser., vol. 81, edited by M. Engebretson, K. Takahashi, and M. Scholer, p. 25, AGU, Washington, D. C., 1994.

Anderson, B. J., and M. J. Engebretson, Relative intensity of toroidal and compressional Pc3-4 wave power in the dayside outer magnetosphere, J. Geophys. Res., 100, 9591, 1995.

Anderson, B. J., M. J. Engebretson, and L. J. Zanetti, Distortion effects in spacecraft observations of MHD toroidal standing waves: Theory and observations, J. Geophys. Res., 94, 13,425, 1989.

Anderson, B. J., M. J. Engebretson, S. P. Rounds, L. J. Zanetti, and T. A. Potemra, A statistical study of Pc3-5 pulsations observed by the AMPTE/ CCE magnetic fields experiment, 1, Occurrence distributions, J. Geophys. Res., 95, 10,495, 1990.

Arthur, C. W., and R. L. McPherron, Interplanetary magnetic field conditions associated with synchronous orbit: Observations of Pc3 magnetic pulsations, J. Geophys. Res., 82, 5138, 1977.

Atkinson, C. T., and T. Watanabe, Surface waves on the magnetospheric boundary as possible origin of long period micropulsations, Earth Planet. Sci. Lett., 1, 89, 1966.

Baransky, L. N., S. P. Belokris, Y. E. Borovkov, M. B. Gokhberg, E. N. Fedorov, and C. A. Green, Restoration of the meridional structure of geomagnetic pulsation fields from gradient measurements, Planet. Space Sci., 37, 859, 1989.

Chen, L., and A. Hasegawa, A theory of long-period magnetic pulsations, 1, Steady state excitation of field line resonance, J. Geophys. Res., 79, 1024, 1974a.

Chen, L., and A. Hasegawa, A theory of long-period magnetic pulsations, 2, Impulse excitation of surface eigenmode, J. Geophys. Res., 79, 1033, 1974b.

Chi, P. I., and C. T. Russell, Phase skipping and poynting flux of continuous pulsations, J. Geophys. Res., 103, 29,479, 1998.

Collet, E., Polarized Light: Fundamentals and Applications, Marcel Dekker, New York, 1993.

Cross, R., An Introduction to Alfven Waves, IOP Publ., Bristol, England, 1988.

Dungey, J. W., Hydromagnetic waves, in Geophysics, the Earth's Environment, edited by C. DeWitt, J. Hieblot, and A. Leleau, Gordon and Breach, Newark, N. J., 1963.

Engebretson, M. J., L. J. Zanetti, T. A. Potemra, W. Baumjohann, H. Lühr, 
and M. H. Acuna, Simultaneous observation of Pc3-4 pulsations in the solar wind and in the Earth's magnetosphere, J. Geophys. Res., 92, 10,053, 1987.

Fenrich, F. R., J. C. Samson, G. Sofko, and R. A. Greenwald, ULF highand low-m field line resonances observed with the Super Dual Auroral Radar Network, J. Geophys. Res., 100, 21,535, 1995.

Glassmeier, K. H., H. Volpers, and W. Baumjohann, Ionospheric joule dissipation as a damping mechanism for high latitude ULF pulsations: Observational evidence, Planet. Space Sci., 32, 1463, 1984.

Greenstadt, E. W., and J. V. Olson, A contribution to ULF activity in the Pc 3-4 range correlated with IMF radial orientation, J. Geophys. Res., 82, 4991, 1977.

Greenstadt, E. W., M. M. Mellott, R. L. McPherron, C. T. Russell, H. J. Singer, and D. J. Knecht, Transfer of pulsation-related wave activity across the magnetopause: Observations of corresponding spectra by ISEE-1 and ISEE-2, Geophys. Res. Lett., 10, 659, 1983.

Gul'elmi, A. V., Theory of hydromagnetic sounding of plasma concentration in the exosphere, Geomagn. Aeron., 7, 357, 1967.

Harrold, B. G., and J. C. Samson, Standing ULF modes of the magnetosphere: A theory, Geophys. Res. Lett., 19, 1811, 1992.

Hoppe, M., C. T. Russell, L. A. Frank, T. E. Eastman, and E. W. Greenstadt, Upstream hydromagnetic waves and their association with backstreaming ion populations: ISEE-1 and 2 observations, J. Geophys. Res., 86, 4471, 1981.

Inhester, B., Numerical modeling of hydromagnetic wave coupling in the magnetosphere, J. Geophys. Res., 92, 4751, 1987.

Itonaga, M., T.-I. Kitamura, O. Saka, H. Tachihara, M. Shinohara, and A. Yoshikawa, Discrete spectral structure of low-latitude and equatorial Pi2 pulsation, J. Geomagn. Geoelectr., 44, 253, 1992.

Khurana, K. K., and M. G. Kivelson, Ultralow frequency MHD waves in Jupiter's middle magnetosphere, J. Geophys. Res., 94, 5241, 1989.

Kim, K.-H., and K. Takahashi, Statistical analysis of compressional Pc3-4 pulsations observed by AMPTE CCE at $L=2-3$ in the dayside magnetosphere, J. Geophys. Res., 104, 4539, 1999.

Kim, K.-H., K. Takahashi, and B. J. Anderson, Ground-satellite coherence analysis of Pc3 pulsations, J. Geophys. Res, 103, 11,755, 1998.

Kitamura, T., and J. A. Jacobs, Determination of the magnetospheric plasma density by the use of long-period geomagnetic micropulsations, Geomagn. Geoelec., 20, 33, 1968.

Kivelson, M. G., and D. J. Southwood, Resonant ULF waves: A new interpretation, Geophys. Res. Lett., 12, 49, 1985.

Kivelson, M. G., and D. J. Southwood, Coupling of global magnetospheric MHD eigenmodes to field line resonances, J. Geophys. Res., 91, 4345, 1986.

Kivelson, M. G., J. Etcheto, and J. G. Trotignon, Global compressional oscillations of the terrestrial magnetosphere: The evidence and a model, J. Geophys. Res., 89, 9851, 1984.

Kivelson, M. G., M. Cao, R. L. McPherron, and R. J. Walker, A possible signature of magnetic cavity mode oscillations in ISEE spacecraft observations, J. Geomagn. Geoelectr., 49, 1079, 1997.

Krauss-Varban, D., and V. L. Patel, Numerical analysis of the coupled hydromagnetic equations in the magnetosphere, J. Geophys. Res., 93, 9721, 1988.

Lee, D.-H., Dynamics of MHD wave propagation in the low-latitude magnetosphere, J. Geophys. Res., 101, 15,371, 1996.

Lee, D.-H., and K. Kim, Compressional MHD waves in the magnetosphere: A new approach, J. Geophys. Res., 104, 12,379, 1999.

Lee, D.-H., and R. L. Lysak, Impulsive excitation of ULF waves in the three dimensional dipole model: The initial results, J. Geophys. Res., 96, $3479,1991$.

Lee, D.-H., and R. L. Lysak, MHD waves in a three-dimensional dipolar magnetic field: A search for Pi2 pulsations, J. Geophys. Res., 104, 28,691, 1999

Lee, D.-H., R. L. Lysak, and Y. Song, Field line resonances in a nonaxisymmetric magnetic field, J. Geophys. Res., 105, 10,703, 2000.

Lin, C. A., L. C. Lee, and Y. J. Sun, Observations of Pi2 pulsations at a very low latitude $(\mathrm{L}=1.06)$ station and magnetospheric cavity resonances, J. Geophys. Res., 96, 21,105, 1991.

Mann, I. R., A. N. Wright, K. J. Mills, and V. M. Nakariakov, Excitation of magnetospheric waveguide modes by magnetosheath flows, J. Geophys. Res., 104, 333, 1999.

Menk, F. W., C. L. Waters, and B. J. Fraser, Field line resonances and waveguide modes at low latitudes, 1, Observations, J. Geophys. Res., $105,7747,2000$

Mills, K. J., A. N. Wright, and I. R. Mann, Kelvin-Helmholtz driven modes of the magnetosphere, Phys. Plasmas, 6, 4070, 1999.

Mitchell, D. G., M. J. Engebretson, D. J. Williams, C. A. Cattell, and R. Lundin, Pc5 pulsations in the outer dawn magnetosphere seen by ISEE 1 and 2, J. Geophys. Res., 95, 967, 1990.

Mond, M., E. Hameiri, and P. N. Hu, Coupling of magnetohydrodynamic waves in inhomogeneous magnetic field configurations, J. Geophys. Res., 95, 89, 1990

Mozer, F. S., R. B. Torbert, U. V. Fahleson, C.-G. Falthammar, A. Gonfalone, and A. Pedersen, Measurements of quasi-static and low-frequency electric fields with spherical double probes on the ISEE-1 spacecraft, IEEE Trans. Geosci. Electron., GE-16, 258, 1978.

Obayashi, T., and J. A. Jacobs, Geomagnetic pulsations and the Earth's outer atmosphere, Geophys. J., 1, 53, 1958.

Odera, T. J., D. Van Swol, C. T. Russell, Simultaneous observation of Pc 3, 4 pulsations in the magnetosphere and at multiple ground stations, Solar Wind Sources of Magnetospheric Ultra-Low Frequency Waves, Geophys. Monogr. Ser., vol. 81, edited by M. Engebretson, K. Takahashi, and M. Scholer, p. 311, AGU, Washington, D. C., 1994.

Olson, J. V., and J. C. Samson, On the detection of the polarization states of Pc micropulsations, Geophys. Res. Lett., 6, 413, 1979.

Orr, D., Magnetic pulsations within the magnetosphere: A review, J. Atmos. Terr. Phys., 35, 1, 1973.

Radoski, H. R., Magnetic toroidal resonances and vibrating field lines, J. Geophys. Res., 71, 1891, 1966

Radoski, H. R., A theory of latitude dependent geomagnetic micropulsations: The asymptotic fields, J. Geophys. Res., 79, 595, 1974.

Rickard, G. J., and A. N. Wright, ULF pulsations in a magnetospheric waveguide: Comparison of real and simulated satellite data, J. Geophys. Res., 100, 3531, 1995.

Ruohoniemi, J. M., R. A. Greenwald, K. B. Baker, and J. C. Samson, HF radar observations of Pc5 field line resonances in the midnight/early morning MLT sector, J. Geophys. Res., 96, 15,697, 1991.

Russell, C. T., The ISEE-1 and ISEE-2 fluxgate magnetometers, IEEE Trans. Geosci. Electron., GE-16, 239, 1978.

Samson, J. C., and B. G. Harrold, Field line resonances associated with MHD waveguides in the magnetosphere, Geophys. Res. Lett., 19, 441, 1992.

Samson, J. C., and J. V. Olson, Data-adaptive polarization filters for multichannel geophysical data, Geophysics, 46, 1423, 1981.

Samson, J. C., J. A. Jacobs, and G. Rostoker, Latitude-dependent characteristics of long-period geomagnetic pulsations, J. Geophys. Res., 76, 3675, 1971.

Samson, J. C., R. A. Greenwald, J. M. Ruohoniemi, T. J. Hughes, and D. D. Wallis, Magnetometer and radar observations of magnetohydrodynamic cavity modes in the Earth's magnetosphere, Can. J. Phys., 69, 929, 1991.

Samson, J. C., C. L. Waters, F. W. Menk, and B. J. Fraser, Fine structure in the spectra of low latitude field line resonances, Geophys. Res. Lett., 22, $2111,1995$.

Singer, H. J., C. T. Russell, M. G. Kivelson, T. A. Fritz, and W. Lennartsson, Satellite observations of the spatial extent of Pc 3, 4, 5 pulsations near the magnetospheric equator, Geophys. Res. Lett., 6, 889, 1979.

Singer, H. J., W. J. Hughes, and C. T. Russell, Standing hydromagnetic waves observed by ISEE 1 and 2: Radial extent and harmonic, J. Geophys. Res., 87, 3519, 1982.

Southwood, D. J., The hydromagnetic stability of the magnetospheric boundary, Planet. Space Sci., 16, 857, 1968.

Southwood, D. J., Some features of field line resonances in the magnetosphere, Planet. Space Sci., 17, 483, 1974.

Stellmacher, M., K.-H. Glassmeier, R. L. Lysak, and M. G. Kivelson, Field line resonances in discretized magnetospheric models: an artifact study, Ann. Geophys., 15, 614, 1997.

Stix, T. H., The Theory of Plasma Waves, McGraw-Hill, New York, 1962. Sutcliffe, P. R., and K. Yumoto, On the cavity mode nature of low latitude Pi2 pulsations, J. Geophys. Res., 96, 1543, 1991.

Takahashi, K., ULF waves in the magnetosphere, Rev. Geophys., 29, 1066, 1991.

Takahashi, K., and R. L. McPherron, Multispacecraft observations of the harmonic structure of Pc3-4 magnetic pulsations, J. Geophys. Res., 89, 6758, 1984 .

Takahashi, K., B. J. Anderson, and R. J. Strangeway, AMPTE CCE observations of Pc 3-4 pulsations at $L=2-6$, J. Geophys. Res., 95, 17,179, 1990.

Tamao, T., Transmission and coupling resonance of hydromagnetic disturbances on the non-uniform Earth's magnetosphere, Tohoku Geophys. J., 17, 43, 1966.

Troitskaya, V. A., Early ground based approach to hydromagnetic diagnostics of outer space, Discovery of the magnetosphere, in Discovery of the Magnetosphere, Hist. Geophys. Ser., vol. 7, edited by C. S. Gillmor and J. R. Spreiter, p. 221, AGU, Washington, D. C., 1997.

Troitskaya, V. A., T. A. Plyasova-Bakunina, and A. V. Gul'yel'mi, Relationship between Pc 2-4 pulsations and the interplanetary magnetic field, Dokl. Akad. Nauk. SSSR, 197, 1312, 1971.

Tsurutani, B. T., and P. Rodriguez, Upstream waves and particles: An overview of ISEE results, J. Geophys. Res., 86, 4319, 1981. 
Walker, A. D. M., J. M. Ruohoniemi, K. D. Baker, R. A. Greenwald, and J. C. Samson, Spatial and temporal behavior of ULF pulsations observed by the Goose Bay HF radar, J. Geophys. Res., 97, 12,187, 1992.

Waters, C. L., F. W. Menk, and B. J. Fraser, The resonance structure of low latitude Pc 3 geomagnetic pulsations, Geophys. Res. Lett., 18, 2293, 1991

Waters, C. L., J. C. Samson, and E. F. Donovan, Variation of plasmatrough density derived from magnetospheric field line resonances, J. Geophys. Res., 101, 24,737, 1996.

Waters, C. L., B. G. Harrold, F. W. Menk, J. C. Samson, and B. J. Fraser, ULF waveguide mode waves at low latitudes, 2, A model, J. Geophys. Res., 105, 7763, 2000.

Waters, C. L., ULF Resonance structure in the magnetosphere, Adv. Space Res., 25(7/8), 1541, 2000.

Wright, A. N., Dispersion and wave coupling in inhomogeneous MHD waveguides, J. Geophys. Res., 99, 159, 1994.

Zhu, X., and M. G. Kivelson, Analytic formulation and quantitative solu- tions of the coupled ULF wave problem, J. Geophys. Res., 93, 8602, 1988

Zhu, X., and M. G. Kivelson, Global mode ULF pulsations in a magnetosphere with a nonmonotonic Alfven velocity profile, J. Geophys. Res., 94, 1479, 1989.

B. J. Anderson and K. Takahashi, Applied Physics Laboratory, Johns Hopkins University, 11000 Johns Hopkins Road, Laurel, MD, 20723-6099, USA.

D.-H. Lee, Department of Astronomy and Space Science, Kyung Hee University, Yongin, Kyunggi, 449-701 Korea.

C. L. Waters, School of Mathematical and Physical Sciences and CRC for Satellite Systems, University of Newcastle, University Drive, Callaghan, NSW 2308, Australia. (physpuls8@cc.newcastle.edu.au) 


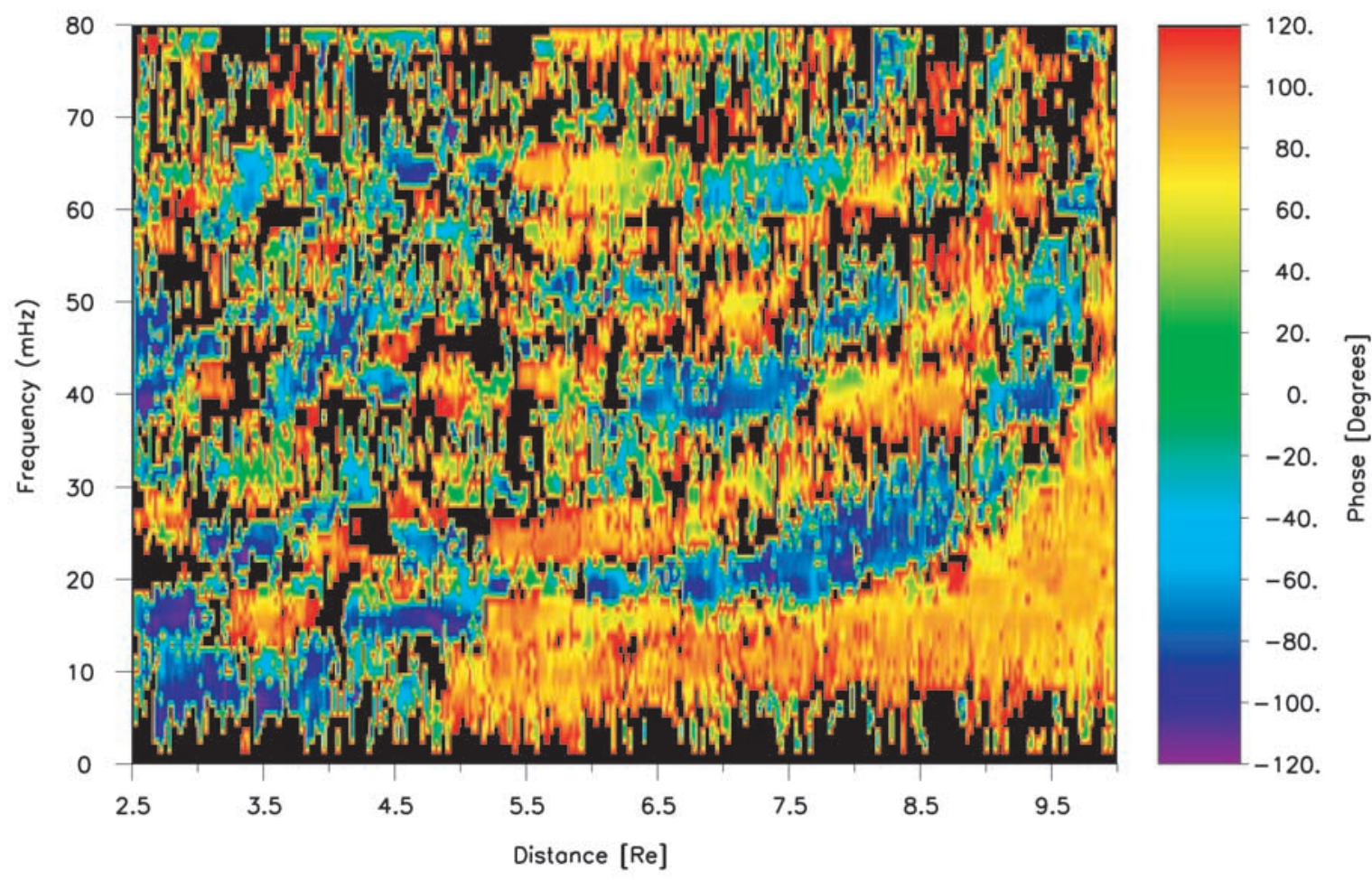

Figure 8. The cross phase of the electric field $\left(e_{y}\right)$ and magnetic field $\left(b_{z}\right)$ data from the 3-D MHD model data. At constant frequency (e.g. $39 \mathrm{mHz}$ ), the cross phase alternates with radial distance between $\pm 90^{\circ}$, as expected from of a standing wave.

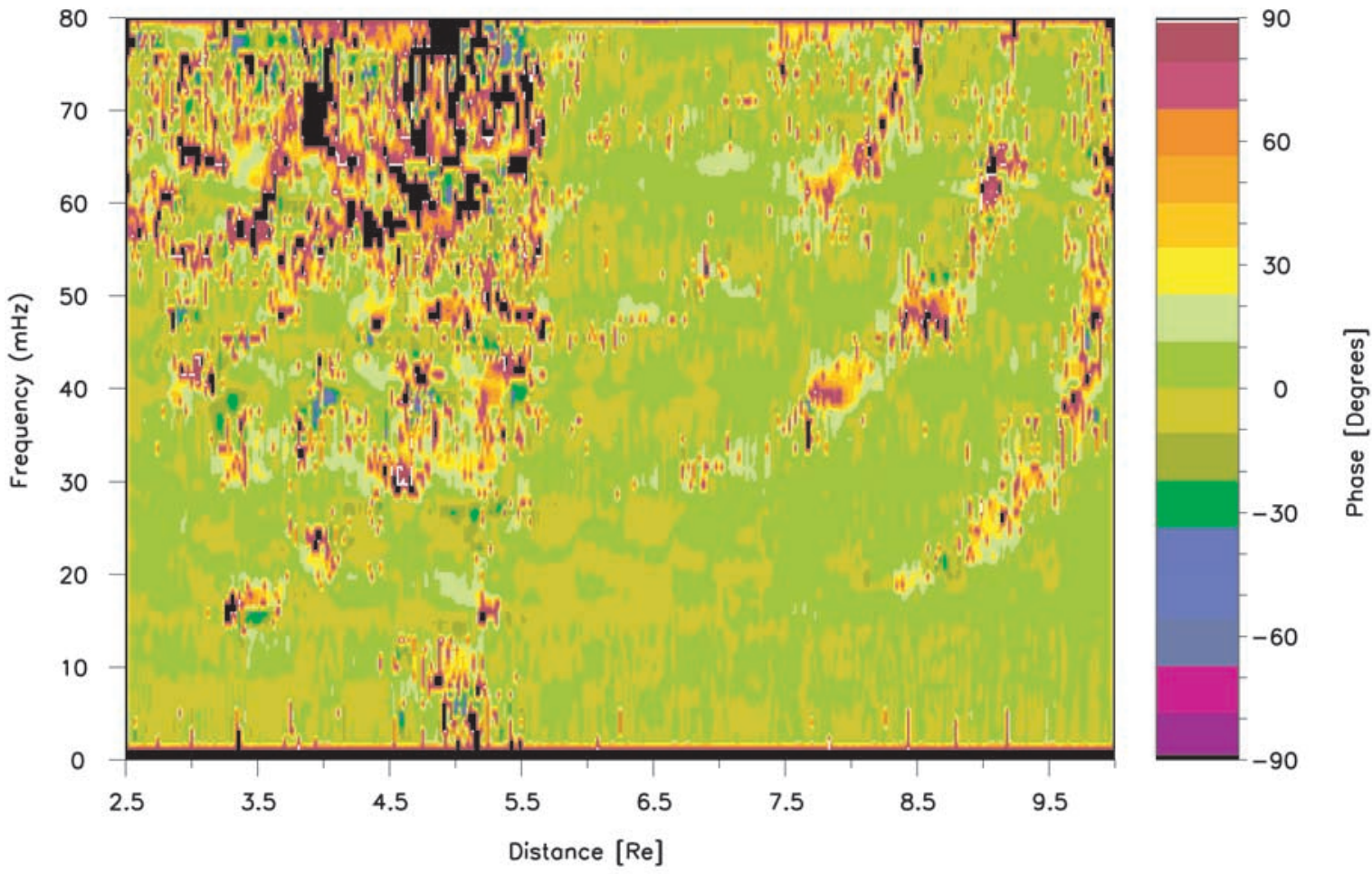

Figure 9. The cross phase of $b_{z}$ obtained from two simulated spacecraft trajectories (equatorial, radial pass and 1100 MLT) through the 3-D MHD model. This FMR detection method is based in Figure 6 where the phase is seen to change rapidly across the FMR nodes in $b_{z}$. Note for example, the large cross phase for $39 \mathrm{mHz}$ near $8 R_{E}$. The simulated spacecraft were given a trajectory speed similar to AMPTE/CCE. 


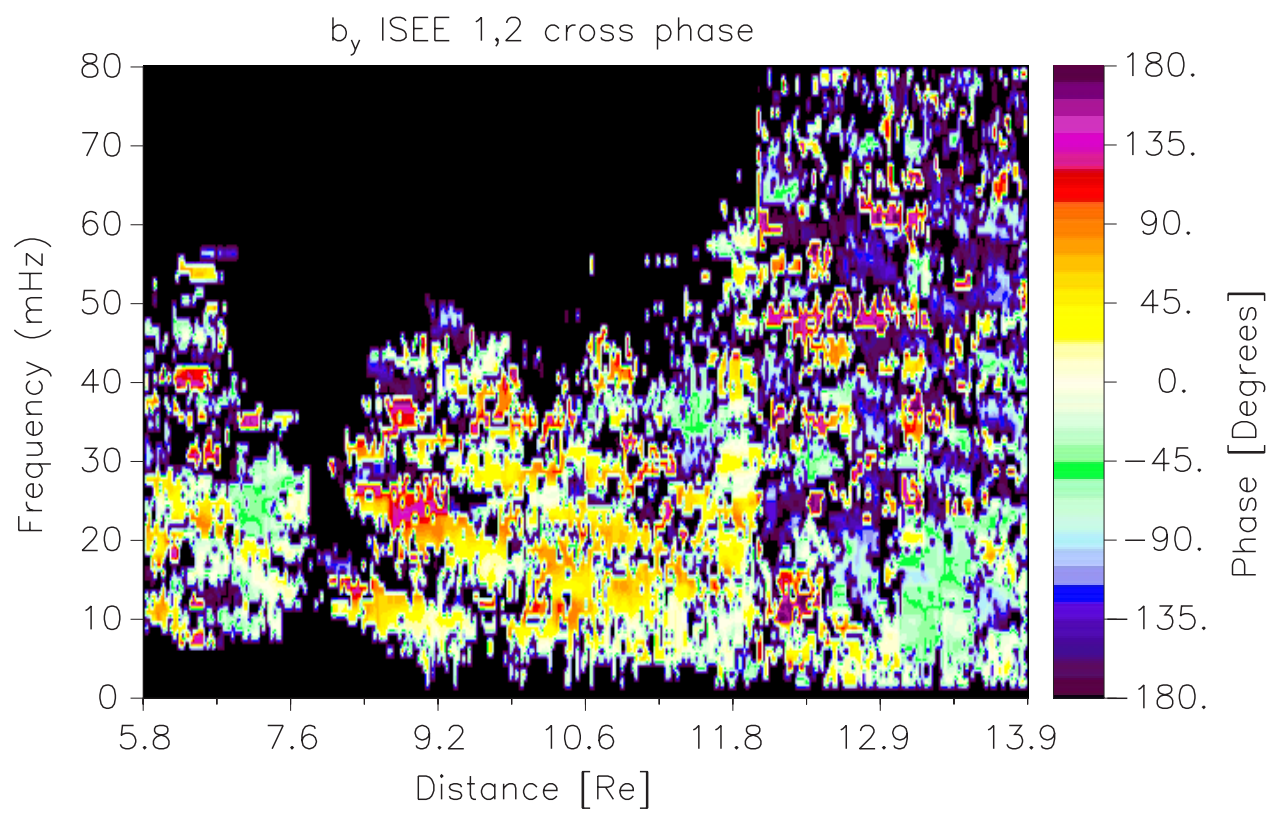

(a)

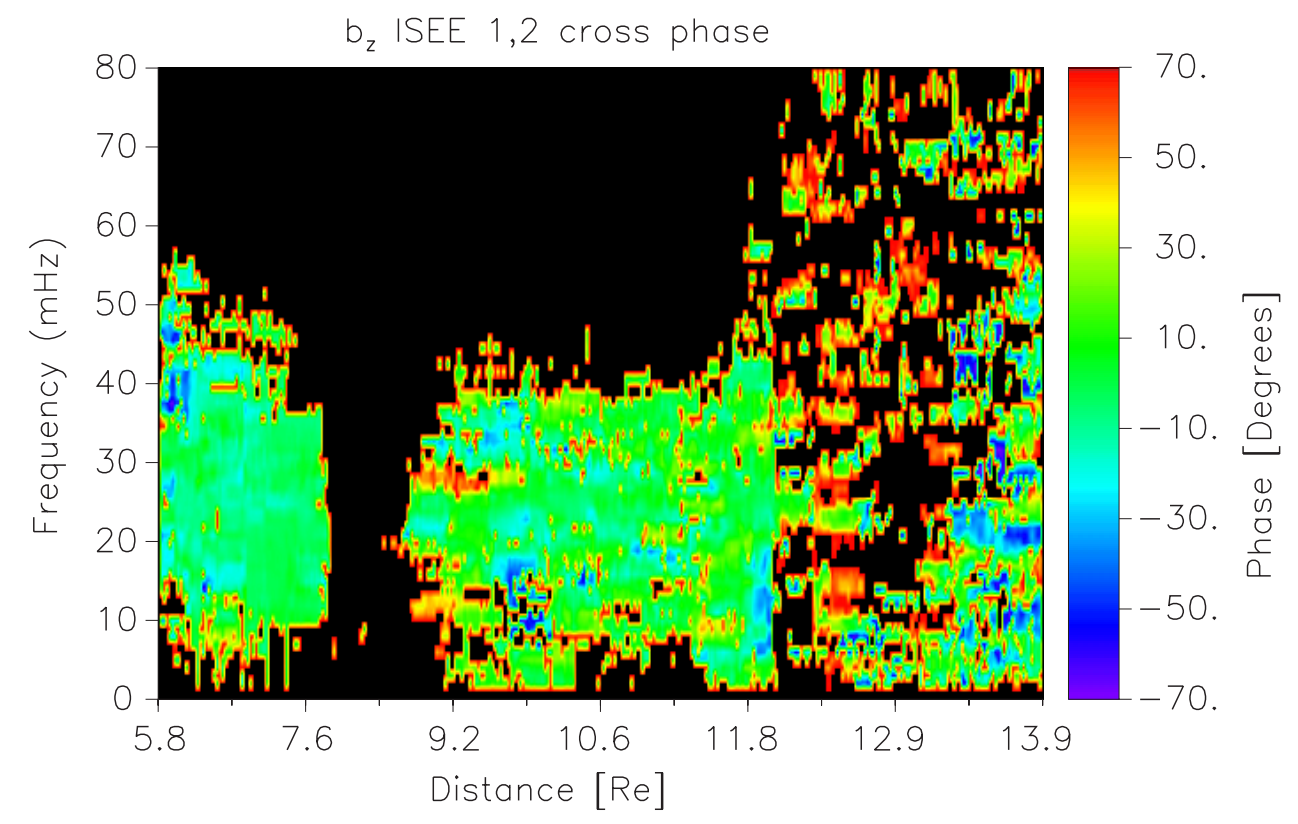

(b)

Figure 13. The cross phase spectra of ISEE 1 and 2 data for 1800-2400 UT, 24 November 1977. (a) The $b_{y}$ components showing multiharmonic FLRs. (b) The $b_{z}$ component data with a change in scale of the phase to highlight the smaller cross phase. The spacecraft entered the magnetosphere at $12.4 R_{E}$. 


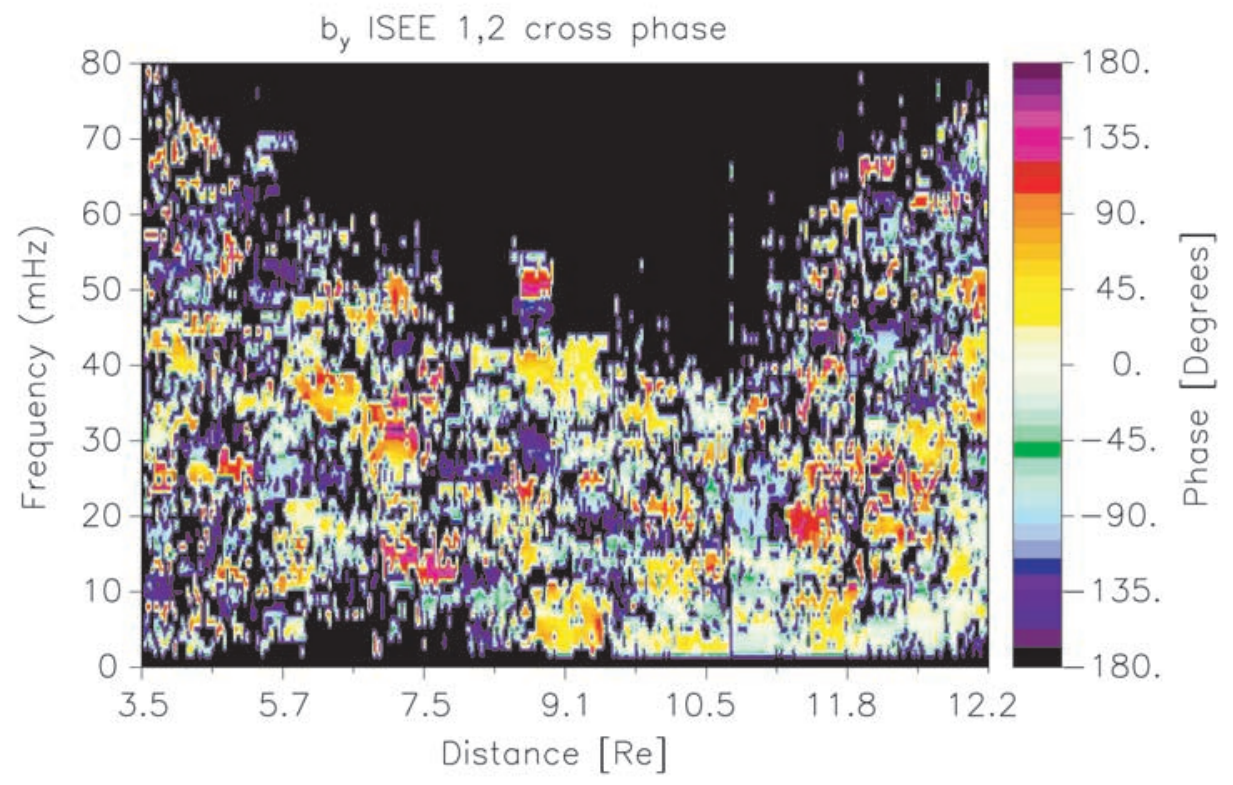

(a)

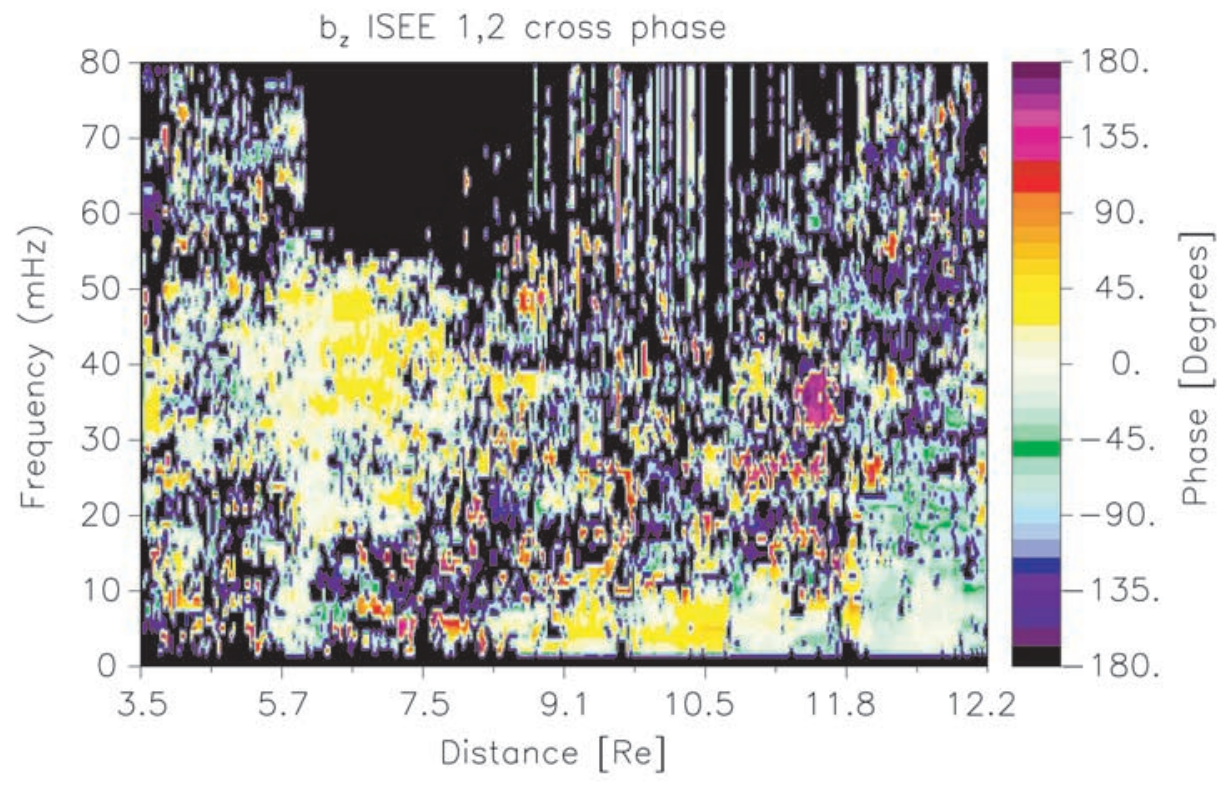

(b)

Figure 15. Cross phase of data from ISEE 1 and 2 for 0200-0730 UT, 14 February 1978: (a) cross phase of ISEE 1 and 2, azimuthal $\left(b_{\mathrm{y}}\right)$ data and (b) cross phase of ISEE 1 and 2, $b_{z}$ data. Note the lack of consistent, $\mathrm{b}_{\mathrm{z}}$ cross-phase values for frequencies greater than $10 \mathrm{mHz}$ and for distances greater than $8 R_{E}$. 